\title{
ON A FLOW OF OPERATORS ASSOCIATED TO VIRTUAL PERMUTATIONS
}

\author{
JOSEPH NAJNUDEL AND ASHKAN NIKEGHBALI
}

\begin{abstract}
In [3, Kerov, Olshanski and Vershik introduce the so-called virtual permutations, defined as families of permutations $\left(\sigma_{N}\right)_{N \geq 1}, \sigma_{N}$ in the symmetric group of order $N$, such that the cycle structure of $\sigma_{N}$ can be deduced from the structure of $\sigma_{N+1}$ simply by removing the element $N+1$. The virtual permutations, and in particular the probability measures on the corresponding space which are invariant by conjugation, have been studied in a more detailed way by Tsilevich in 13 and [14. In the present article, we prove that for a large class of such invariant measures (containing in particular the Ewens measure of any parameter $\theta \geq 0$ ), it is possible to associate a flow $\left(T^{\alpha}\right)_{\alpha \in \mathbb{R}}$ of random operators on a suitable functional space. Moreover, if $\left(\sigma_{N}\right)_{N>1}$ is a random virtual permutation following a distribution in the class described above, the operator $T^{\alpha}$ can be interpreted as the limit, in a sense which has to be made precise, of the permutation $\sigma_{N}^{\alpha_{N}}$, where $N$ goes to infinity and $\alpha_{N}$ is equivalent to $\alpha N$. In relation with this interpretation, we prove that the eigenvalues of the infinitesimal generator of $\left(T^{\alpha}\right)_{\alpha \in \mathbb{R}}$ are equal to the limit of the rescaled eigenangles of the permutation matrix associated to $\sigma_{N}$.
\end{abstract}

\section{IntroduCtion}

A large part of the research in random matrix theory comes from the problem of finding the possible limit distributions of the eigenvalues of a given ensemble of random matrices, when the dimension goes to infinity. Many different ensembles have been studied (see, for example, Mehta [7]), the most classical one is the so-called Gaussian Unitary Ensemble (GUE), where the corresponding random matrix is hermitian, the diagonal entries are standard gaussian variables and the real and the imaginary part of the entries above the diagonal are centered gaussian variables of variance $1 / 2$, all these variables being independent. Another well-known ensemble is the Circular Unitary Ensemble (CUE), corresponding to a random matrix which follows the Haar measure on a finite-dimensional unitary group. A remarkable phenomenon which happens is the so-called universality property: there are some particular point processes which appear as the limit (after suitable scaling) of the distribution of the eigenvalues (or eigenangles) associated with a large class of random matrix ensembles, this limit being independent of the detail of the model which is considered. For example, after scaling, the small eigenvalues of the GUE and the small eigenangles of the CUE converge to the same process, called determinantal process with sine kernel, and appearing as the limit of a number of other models of random matrices. This process is a point process on the real line, such that informally, for $x_{1}, \ldots, x_{n} \in \mathbb{R}$, the probability to have a point in the neighborhood of $x_{j}$ for all $j \in\{1, \ldots, k\}$ is proportional to the determinant of the matrix $\left(K\left(x_{j}, x_{k}\right)\right)_{1 \leq j, k \leq n}$,

Date: October 1, 2018. 
where the kernel $K$ is given by the formula:

$$
K(x, y)=\frac{\sin (\pi(x-y))}{\pi(x-y)} .
$$

Another point process which enjoys some universality properties is the determinantal process with Airy kernel, which is involved in the distribution of the largest eigenvalues of the GUE, and which is defined similarly as the determinantal process with sine kernel, except that the kernel $K$ is now given by:

$$
K(x, y)=\frac{\operatorname{Ai}(x) \operatorname{Ai}^{\prime}(y)-\operatorname{Ai}(y) \operatorname{Ai}^{\prime}(x)}{x-y}
$$

where Ai denotes the Airy function. The phenomenon of universality is not well-explained in its full generality: a possible way the have a good understanding of the corresponding point processes is to express them as the set of eigenvalues of some universal infinite-dimensional random operators, and to prove that these operators are the limits, in a sense which needs to be made precise, of the classical random matrix ensembles. Such a construction has been done by Ramirez, Rider and Virág [12, where, as a particular case, the authors express a determinantal process with Airy kernel as the set of the eigenvalues of a random differential operator on a functional space. Moreover, this infinite-dimensional operator is naturally interpreted as the limit of an ensemble of tridiagonal matrices, which have the same eigenvalue distribution as the GUE. However, this operator is not directly constructed from GUE (or another classical model as CUE), and the reason of its universal properties are not obvious. On the other hand, an operator whose eigenvalues form a determinantal process with sine kernel, and which is related in a natural way to a classical random matrix model, has not been yet defined: a construction which seems to be very promising involves the interpretation of the sine kernel process as a function of a stochastic process called the Brownian carousel and constructed by Valkó and Virág [15]. Now, despite the fact that it seems to be particularly difficult to associate, in a natural way, an infinite-dimensional operator to the most classical matrix ensembles, we shall prove, in the present paper, that such a construction is possible and very explicit for a large class of ensembles of permutations matrices. These ensembles, and some of their generalizations, have already be studied by several authors (see, for example, Evans [2] and Wieand [16]), including the authors of the present paper (see [8]). An important advantage of permutations matrices is the fact that their eigenvalues can directly be expressed in function of the size of the cycles of the corresponding permutations. Hence, it is equivalent to study these matrices or to deal with the corresponding cycle structure. Another advantage is the existence of a quite convenient way to define models of permutation matrices in all the different dimensions, on the same probability space, which gives a meaning to the notion of almost sure convergence when the dimension goes to infinity (a rather unusual situation in random matrix theory). This also gives the possibility to define an infinite-dimensional limit model, which will be explicitly constructed in this paper. Once the permutations and their matrices are identified, the main objects involved in our construction are the so-called virtual permutations, first introduced by Kerov, Olshanski and Vershik in [3], and further studied by Tsilevich in [13] and 14]. A virtual permutation can be defined as follows: it is a sequence $\left(\sigma_{N}\right)_{N \geq 1}$ of permutations, $\sigma_{N}$ being of order $N$, and such that the cycle structure of $\sigma_{N}$ is obtained from the cycle structure of $\sigma_{N+1}$ by simply removing the element $N+1$. Then, for all $\theta \geq 0$, it is possible to define a unique probability 
measure on the space of virtual permutations, such that for all $N \geq 0$, its image by the $N$-th coordinate is equal to the Ewens measure on the symmetric group of order $N$, with parameter $\theta$. The Ewens measures on the space of virtual permutations are particular cases of the so-called central measures, studied in [14], and defined as the probability measures which are invariant by conjugation with any permutation of finite order (see below for the details). The central measures are completely characterized in [14], using the properties of exchangeable partitions, stated by Kingman in [4, [5] and [6] (see also the course by Pitman [11]). The following remarkable statement holds: if the distribution of a random virtual permutation $\left(\sigma_{N}\right)_{N \geq 1}$ is a central measure, and if for all $N \geq 1,\left(l_{k}^{(N)}\right)_{k \geq 1}$ denotes the decreasing sequence of cycle lengths (completed by zeros) of the permutation $\sigma_{N}$, then for all $k \geq 1$, the sequence $\left(l_{k}^{(N)} / N\right)_{N \geq 1}$ converges almost surely to a limit random variable $\lambda_{k}$. By using Fatou's lemma, one immediately deduces that almost surely, the random sequence $\left(\lambda_{k}\right)_{k \geq 1}$ is nonnegative and decreasing, with:

$$
\sum_{k \geq 1} \lambda_{k} \leq 1
$$

If the distribution of $\left(\sigma_{N}\right)_{N \geq 1}$ is the Ewens measure of parameter $\theta \geq 0$, then $\left(\lambda_{k}\right)_{k \geq 1}$ is a Poisson-Dirichlet process with parameter $\theta$ (if $\theta=0$, then $\lambda_{1}=1$ and $\lambda_{k}=0$ for $k \geq 1$ ). This convergence of the renormalized cycle lengths can be translated into a statement on random matrices. Indeed, to any virtual permutation following a central measure, one can associate a sequence $\left(M_{N}\right)_{N \geq 1}$ of random permutation matrices, $M_{N}$ being of dimension $N$. If for $N \geq 1, X_{N}$ denotes the point process of the eigenangles of $M_{N}$, multiplied by $N$ and counted with multiplicity, then $X_{N}$ converges almost surely to the limit process $X_{\infty}$ defined as follows:

- $X_{\infty}$ contains, for all $k \geq 1$ such that $\lambda_{k}>0$, each non-zero multiple of $2 \pi / \lambda_{k}$.

- The multiplicity of any non-zero point $x$ of $X_{\infty}$ is equal to the number of values of $k$ such that $\lambda_{k}>0$ and $x$ is multiple of $2 \pi / \lambda_{k}$.

- The multiplicity of zero is equal to the number of values of $k \geq 1$ such that $\lambda_{k}>0$ if $\sum_{k \geq 1} \lambda_{k}=1$, and to infinity if $\sum_{k \geq 1} \lambda_{k}<1$.

This convergence has to be understood in the following way: for all functions $f$ from $\mathbb{R}$ to $\mathbb{R}_{+}$, continuous with compact support, the sum of $f$ at the points of $X_{N}$, counted with multiplicity, tends to the corresponding sum for $X_{\infty}$ when $N$ goes to infinity. In the case of the Ewens probability measure with parameter $\theta>0$, a detailed proof of this result is given by the present authors in [8], in a more general context. Once the almost sure convergence of the rescaled eigenangles is established, one can naturally ask the following question: is it possible to express the limit point process as the spectrum of a random operator associated to the virtual permutation which is considered? The main goal of this article is to show that the answer is positive. More precisely, we prove that in a sense which can be made precise, for almost every virtual permutation following a central probability distribution, for all $\alpha \in \mathbb{R}$, and for all sequences $\left(\alpha_{N}\right)_{N \geq 1}$ such that $\alpha_{N}$ is equivalent to $\alpha N$ for $N$ going to infinity, $\sigma_{N}^{\alpha_{N}}$ converges to an operator $T^{\alpha}$, depending on $\left(\sigma_{N}\right)_{N \geq 0}$, on $\alpha$ but not on the choice of $\left(\alpha_{N}\right)_{N \geq 1}$. The flow of operators $\left(T^{\alpha}\right)_{\alpha \in \mathbb{R}}$ (which almost surely satisfies $T^{\alpha+\beta}=T^{\alpha} T^{\beta}$ for all $\alpha, \beta \in \mathbb{R}$ ) is defined on a functional space which will be constructed later, and admits almost surely an infinitesimal generator $U$. Moreover, we prove that the spectrum of $i U$ is exactly given by the limit point process $X_{\infty}$ constructed above. This spectral interpretation of such a 
limit point process suggests that it is perhaps possible to construct similar objects for more classical matrix models, despite the discussion above about the difficulty of this problem. Indeed, in [10], Olshanski and Vershik characterize the measures on the space of infinite dimensional hermitian matrices, which are invariant by conjugation with finite-dimensional unitary matrices. These central measures enjoy the following property: if a random infinite matrix follows one of them, then after suitable scaling, the point process of the extreme eigenvalues of its upper-left finite-dimensional submatrice converges almost surely to a limit point process when the dimension of this submatrice tends to infinity, similarly as for the case of virtual permutations, where $X_{N}$ tends to $X_{\infty}$. By the Cayley transform, the sequence of the upper-left submatrices of an infinite hermitian matrix is mapped to a sequence $\left(M_{N}\right)_{N \geq 1}$ of unitary matrices, such that the matrix $M_{N}$ can be deduces from $M_{N+1}$ by an projective map, explicitly described by Neretin [9]. Among the central measures described in [10], there exists a unique measure for which all its projections on the finite-dimensional unitary groups are equal to Haar measure. In [1] Borodin and Olshanski study the hermitian version of this measure, and a family of generalizations, depending on a complex parameter and called Hua-Pickrell measures. In particular, they prove that the corresponding point process is determantal and compute explicitly its kernel: for the Haar measure, they obtain the image of a sine kernel process by the map $x \mapsto 1 / x$. Moreover, in a forthcoming paper with Bourgade, by using the splitting of unitary matrices as a product of reflections, we define the natural generalization of virtual permutations to sequences of general unitary matrices of increasing dimensions. These sequences are different from the sequences of unitary matrices defined by Neretin, Olshanski and Vershik, in spite of the similarity between the two constructions. In our setting, there exists also a projective limit of the Haar measure, and under this measure, we prove the almost sure convergence of the rescaled eigenangles when the dimension goes to infinity, the limit process being a sine-kernel process. It is also possible to construct a family of measures which has the same finite-dimensional projections as the Hua-Pickrell measures, and to study the almost sure convergence of their rescaled eigenangles. However, contrarily to the case of virtual permutations discussed in the present paper, we are not yet able to interpret the corresponding limit point process as the sequence of eigenvalues of an operator.

The present article is organized as follows: in Section 2, we define the notion of a virtual permutation in a more general setting than it is usually done; in Section 3, we study the central measures in this new setting, generalizing the results by Kerov, Olshanski, Tsilevich and Vershik; in Section 4, we use the results of Section 3 in order to associate, to almost every virtual permutation under a central measure, a flow of transformations of a topological space obtained by completing the set on which the virtual permutations act; in Section 5 , we interpret this flow as a flow of operators on a functional space, and we deduce the construction of the random operator $U$.

\section{Virtual Permutations of General sets}

The virtual permutations are usually defined as the sequences $\left(\sigma_{N}\right)_{N \geq 1}$ such that $\sigma_{N} \in \Sigma_{N}$ for all $N \geq 1$, where $\Sigma_{N}$ is the symmetric group of order $N$, and the cycle structure of $\sigma_{N}$ is obtained by removing $N+1$ from the cycle structure of $\sigma_{N+1}$. In this definition, the order of the integers is involved in an important way, which is not very satisfactory since we are essentially interested in the cycle structure of the permutation, and not particularly in 
the nature of the elements inside the cycles. Therefore, in this section, we present a notion of virtual permutation which can be applied to any set and not only to the set of positive integers. This generalization is possible because of following result:

Proposition 2.1. Let $\left(\sigma_{N}\right)_{N \geq 1}$ be a virtual permutation (in the usual sense). For all finite subsets $I \subset \mathbb{N}^{*}$, and for all $N \geq 1$ larger than any of the elements in $I$, let $\sigma_{I}^{(N)}$ be the permutation of the elements of I obtained by removing the elements outside I from the cycle structure of $\sigma_{N}$. Then $\sigma_{I}^{(N)}$ depends only on $I$ and not on the choice of $N$ majorizing $I$, and one can write $\sigma_{I}^{(N)}=: \sigma_{I}$. Moreover, if $J$ is a finite subset of $\mathbb{N}^{*}$ containing $I$, then $\sigma_{I}$ can be obtained from the cycle structure of $\sigma_{J}$ by removing all the elements of $J \backslash I$.

Proof. Let $N \leq N^{\prime}$ be two integers majorizing $I$. By the classical definition of virtual permutations, $\sigma_{N}$ is obtained by removing the elements strictly larger than $N$ from the cycle structure of $\sigma_{N^{\prime}}$. Hence, $\sigma_{I}^{(N)}$ can be obtained from the cycle structure of $\sigma_{N^{\prime}}$ by removing the elements strictly larger than $N$, and then the elements smaller than or equal to $N$ which are not in $I$. Since all the elements of $I$ are smaller than or equal to $N$, it is equivalent to remove directly all the elements of $\left\{1, \ldots, N^{\prime}\right\}$ outside $I$, which proves that $\sigma_{I}^{\left(N^{\prime}\right)}=\sigma_{I}^{(N)}$. Now, let $J$ be a finite subset of $\mathbb{N}^{*}$ containing $I$, and $N^{\prime \prime}$ an integer which majorizes $J$. The permutation $\sigma_{I}$ is obtained by removing the elements of $\left\{1, \ldots, N^{\prime \prime}\right\} \backslash I$ from the cycle structure of $\sigma_{N^{\prime \prime}}$. It is equivalent to say that $\sigma_{I}$ is obtained from $\sigma_{N^{\prime \prime}}$ by removing the elements of $\left\{1, \ldots, N^{\prime \prime}\right\} \backslash J$, and then the elements of $J \backslash I$, which implies that $\sigma_{I}$ can be obtained by removing the elements of $J \backslash I$ from the cycle structure of $\sigma_{J}$.

We can then define virtual permutations on general sets as follows:

Definition 2.2. A virtual permutation of a given set $E$ is a family of permutations $\left(\sigma_{I}\right)_{I \in \mathcal{F}(E)}$, indexed by the set $\mathcal{F}(E)$ of the finite subsets of $E$, such that $\sigma_{I} \in \Sigma_{I}$, where $\Sigma_{I}$ is the symmetric group of $I$, and such that for all $I, J \in \mathcal{F}(E), I \subset J$, the permutation $\sigma_{I}$ is obtained by removing the elements of $J \backslash I$ from the cycle structure of $\sigma_{J}$.

If $E$ is a finite set, a virtual permutation is essentially a permutation, more precisely, one has the following proposition:

Proposition 2.3. Let $E$ be a finite set and $\sigma$ a permutation of $E$. Then, one can define a virtual permutation $\left(\sigma_{I}\right)_{I \in \mathcal{F}(E)}$ of $E$ as follows: $\sigma_{I}$ is obtained from $\sigma$ by removing the elements of $E \backslash I$ from its cycle structure. Moreover, this mapping is bijective, and the inverse mapping is obtained by associating the permutation $\sigma_{E}$ to any virtual permutation $\left(\sigma_{I}\right)_{I \in \mathcal{F}(E)}$.

This proposition is almost trivial, so we omit the proof.

Notation Because of this result, we will denote the set of virtual permutations of any set $E$ by $\Sigma_{E}$. In the case $E=\mathbb{N}^{*}$, we can immediately deduce the following result from Proposition 2.1:

Proposition 2.4. If $\left(\sigma_{N}\right)_{N \geq 1}$ is a virtual permutation in the classical sense, then with the notation of Proposition [2.1. $\left(\sigma_{I}\right)_{I \in \mathcal{F}\left(\mathbb{N}^{*}\right)}$ is a virtual permutation of $\mathbb{N}^{*}$ in the sense of Definition [2.2. Moreover, the mapping which associates $\left(\sigma_{I}\right)_{I \in \mathcal{F}\left(\mathbb{N}^{*}\right)}$ to $\left(\sigma_{N}\right)_{N \geq 1}$ is bijective: the existence of the inverse mapping is deduced from the fact that $\sigma_{N}=\sigma_{\{1, \ldots, N\}}$.

An example of a virtual permutation defined on an uncountable set is given as follows: if $E$ is a circle, and if for $I \in \mathcal{F}(E), \sigma_{I}$ is the permutation of $I$ containing a unique cycle, 
obtained by counterclockwise enumeration of the points of $I$, then $\left(\sigma_{I}\right)_{I \in \mathcal{F}(E)}$ is a virtual permutation of $E$. In this example, it is natural to say that $\left(\sigma_{I}\right)_{I \in \mathcal{F}(E)}$ contains $E$ itself as a unique cycle. More generally, one can define a cycle structure for any virtual permutation, by the following result:

Proposition 2.5. Let $\left(\sigma_{I}\right)_{I \in \mathcal{F}(E)}$ be a virtual permutation of a set $E$, and let $x, y$ be two elements of E. Then, one of the following two possibilities holds:

- For all $I \in \mathcal{F}(E)$ containing $x$ and $y$, these two elements are in the same cycle of the permutation $\sigma_{I}$;

- For all $I \in \mathcal{F}(E)$ containing $x$ and $y$, these two elements are in two different cycles of $\sigma_{I}$.

The first case defines an equivalence relation on the set $E$.

Proof. Let $I \in \mathcal{F}(E)$ containing $x$ and $y$. By the definition of virtual permutations, it is easy to check that $x$ and $y$ are in the same cycle of $\sigma_{I}$ if and only if $\sigma_{\{x, y\}}$ is equal to the transposition $(x, y)$, these property being independent of the choice of $I$. Moreover, if the first item in Proposition 2.5 holds for $x$ and $y$, and for $y$ and $z$, then the permutation $\sigma_{\{x, y, z\}}$ contains a unique cycle, which implies that the first item holds also for $x$ and $z$.

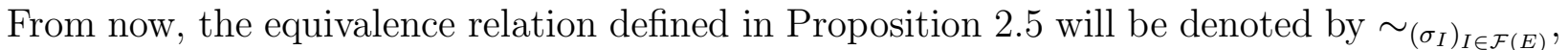
or simply by $\sim$ if no confusion is possible. The corresponding equivalence classes will be called the cycles of $\left(\sigma_{I}\right)_{I \in \mathcal{F}(E)}$. The cycle of an element $x \in E$ will be denoted $\mathcal{C}_{\left(\sigma_{I}\right)_{I \in \mathcal{F}(E)}}(x)$, or simply $\mathcal{C}(x)$. One immediately checks that this notion of cycle is consistent with the classical notion for permutations of finite order. Another notion which should be introduced is the notion of conjugation, involved in the definition of central measures given in Section 3 .

Proposition 2.6. Let $\Sigma_{E}^{(0)}$ be the group of the permutations of $E$ which fix all but finitely many elements of $E$. Then, the group $\Sigma_{E}^{(0)}$ acts on $\Sigma_{E}$ by conjugation, because of the following fact: for all $g \in \Sigma_{E}^{(0)}$, and for all $\sigma=\left(\sigma_{I}\right)_{I \in \mathcal{F}(E)}$, there exists a unique virtual permutation $g \sigma g^{-1}=\left(\sigma_{I}^{\prime}\right)_{I \in \mathcal{F}(E)}$ such that for all $I \in \mathcal{F}(E)$ containing all the points of $E$ which are not fixed by $g, \sigma_{I}^{\prime}=g_{I} \sigma_{I} g_{I}^{-1}$, where $g_{I}$ denotes the restriction of $g$ to $I$. Moreover, the cycles of $g \sigma g^{-1}$ are the images by $g$ of the cycles of $\sigma$.

Proof. Let $\sigma=\left(\sigma_{I}\right)_{I \in \mathcal{F}(E)} \in \Sigma_{E}, g \in \Sigma_{E}^{(0)}$ and denote $E(g)$ the set of points which are not fixed by $g$. For all $I \in \mathcal{F}(E)$, let us define $\sigma_{I}^{\prime}$ as the permutation obtained from $g_{E(g) \cup I} \sigma_{E(g) \cup I} g_{E(g) \cup I}^{-1}$ by removing the elements of $E(g) \backslash I$ from its cycle structure. In other words, the cycle structure of $\sigma_{I}^{\prime}$ can be obtained from the cycle structure of $\sigma_{E(g) \cup I}$ by replacing all the elements by their image by $g$, and then by removing the elements of $E(g) \backslash I$. Now, if $J \in \mathcal{F}(E)$ and $I \subset J$, then the structure of $\sigma_{I}^{\prime}$ can also be obtained from the structure of $\sigma_{E(g) \cup J}$ by removing the elements of $J \backslash(E(g) \cup I)$, by replacing the remaining elements by their image by $g$, and then by removing the elements of $E(g) \backslash I$. Since all the elements of $J \backslash E(g) \cup I$ are fixed by $g$, the order of the two first operations is not important, and then $\sigma_{I}^{\prime}$ is obtained from $\sigma_{E(g) \cup J}$ by replacing the elements by their image by $g$, and then by removing the elements of $(J \cup E(g)) \backslash I$. This implies that $\sigma_{I}^{\prime}$ is obtained from $\sigma_{J}^{\prime}$ by removing the elements of $J \backslash I$ from its cycle structure, in other words, $\left(\sigma_{I}^{\prime}\right)_{I \in \mathcal{F}(E)}$ is a virtual permutation of $E$, which proves the existence of $g \sigma g^{-1}$. Its uniqueness is a direct consequence 
of the fact that all its components corresponding to a set containing $E(g)$ are determined by definition. Since, for $I$ containing $E(g)$, the cycle structure of $\left(g \sigma g^{-1}\right)_{I}$ is obtained from the structure of $\sigma$ by replacing the elements by their image by $g$, it is easy to deduce that the cycles of the virtual permutation $g \sigma g^{-1}$ are the images by $g$ of the cycles of $\sigma$, and that the conjugation is a group action of $\Sigma_{E}^{(0)}$ on $\Sigma_{E}$.

We have so far given the general construction of virtual permutations, and some of their main properties: in Section 3, we introduce probability measures on the space of virtual permutations.

\section{Central measures on general spaces of virtual permutations}

In order to construct a probability measure on the space of virtual permutations of a set $E$, one can expect that it is sufficient to define its images by the coordinate mappings, if these images satisfy some compatibility properties. The following result proves that such a construction is possible if the set $E$ is countable:

Proposition 3.1. Let $E$ be a countable set, let $S(E)$ be the product of all the symmetric groups $\Sigma_{I}$ for $I \in \mathcal{F}(E)$, and let $\mathcal{S}(E)$ be the $\sigma$-algebra on $S(E)$, generated by all the coordinates mappings $\left(\sigma_{I}\right)_{I \in \mathcal{F}(E)} \mapsto \sigma_{J}$ for $J \in \mathcal{F}(E)$. For all $I, J \in \mathcal{F}(E)$ such that $I \subset J$, let $\pi_{J, I}$ be the map from $\Sigma_{J}$ to $\Sigma_{I}$ which removes the elements of $J \backslash I$ from the cycle structure. Let $\left(\mathbb{P}_{I}\right)_{I \in \mathcal{F}(E)}$ be a family of probability measures, $\mathbb{P}_{I}$ defined on the finite set $\Sigma_{I}$, which is compatible in the following sense: for all $I, J \in \mathcal{F}(E)$ such that $I \subset J$, the image of $\mathbb{P}_{J}$ by $\pi_{J, I}$ is $\mathbb{P}_{I}$. Then, there exists a unique probability measure $\mathbb{P}$ on the measurable space $(S(E), \mathcal{S}(E))$ satisfying the following two conditions:

- $\mathbb{P}$ is supported by the set $\Sigma_{E}$ of virtual permutations;

- For all $I \in \mathcal{F}(E)$, the image of $\mathbb{P}$ by the coordinate map indexed by $I$ is equal to the measure $\mathbb{P}_{I}$.

Proof. For every family $\left(I_{k}\right)_{1 \leq k \leq n}$ of elements of $\mathcal{F}(E)$, and for all $J \in \mathcal{F}(E)$ containing $I_{k}$ for all $k$, let $\mathbb{P}_{I_{1}, \ldots, I_{n} ; J}$ be the image of the probability measure $\mathbb{P}_{J}$ by the mapping

$$
\sigma \mapsto\left(\pi_{J, I_{1}}(\sigma), \ldots, \pi_{J, I_{n}}(\sigma)\right)
$$

from $\Sigma_{J}$ to the product space $\Sigma_{I_{1}} \times \cdots \times \Sigma_{I_{n}}$. Then, the following two properties hold:

- Once the sets $\left(I_{k}\right)_{1 \leq k \leq n}$ are fixed, the measure

$$
\mathbb{P}_{I_{1}, \ldots, I_{n}}:=\mathbb{P}_{I_{1}, \ldots, I_{n} ; J}
$$

does not depend on the choice of $J$.

- For $n \geq 2$, the image of $\mathbb{P}_{I_{1}, \ldots, I_{n}}$ by a permutation $\sigma \in \Sigma_{n}$ of the coordinates is equal to $\mathbb{P}_{I_{\sigma(1)}, \ldots, I_{\sigma(n)}}$, and the image by the application removing the last coordinate is $\mathbb{P}_{I_{1}, \ldots, I_{n-1}}$.

These properties can easily be proven by using the compatibility property of the family $\left(\mathbb{P}_{I}\right)_{I \in \mathcal{F}(E)}$. Now, let us observe that a probability measure $\mathbb{P}$ on $(S(E), \mathcal{S}(E))$ satisfies the conditions given in Proposition 3.1 if and only if for all $I_{1}, \ldots, I_{n} \in \mathcal{F}(E)$, the image of $\mathbb{P}$ by the family of coordinates indexed by $I_{1}, \ldots, I_{n}$ is equal to $\mathbb{P}_{I_{1}, \ldots, I_{n}}$ (note that the fact that $E$ is countable is used in this step). The property of compatibility given in the second item above and the Caratheodory extension theorem then imply the existence and the uniqueness of $\mathbb{P}$. 
Remark 3.2. The set $\Sigma_{E}$ is the intersection of the sets $S_{J, K}(E) \in \mathcal{S}(E)$, indexed by the pairs $(J, K) \in \mathcal{F}(E) \times \mathcal{F}(E)$ such that $J \subset K$, and defined as follows: a family $\left(\sigma_{I}\right)_{I \in \mathcal{F}(E)}$ is in $S_{J, K}(E)$ if and only if $\sigma_{J}=\pi_{K, J}\left(\sigma_{K}\right)$. If $E$ is countable, this intersection is countable, and then $\Sigma_{E} \in \mathcal{S}(E)$. However, if $E$ is uncountable, $\Sigma_{E}$ does not seem to be measurable, and we do not know how to construct the measure $\mathbb{P}$ in this case.

An immediate consequence of Proposition 3.1 is the following:

Corollary 3.3. Let $E$ be a countable set, and let $\mathcal{S}_{E}$ be the $\sigma$-algebra on $\Sigma_{E}$, generated by the coordinates mappings $\left(\sigma_{I}\right)_{I \in \mathcal{F}(E)} \mapsto \sigma_{J}$ for $J \in \mathcal{F}(E)$. Let $\left(\mathbb{P}_{I}\right)_{I \in \mathcal{F}(E)}$ be a family of probability measures, $\mathbb{P}_{I}$ defined on $\Sigma_{I}$, which is compatible in the sense of Proposition 3.1. Then, there exists a unique probability measure $\mathbb{P}_{E}$ on the measurable space $\left(\Sigma_{E}, \mathcal{S}_{E}\right)$ such that for all $I \in \mathcal{F}(E)$, the image of $\mathbb{P}_{E}$ by the coordinate indexed by $I$ is equal to $\mathbb{P}_{I}$.

An example of measure on $\Sigma_{E}$ which can be constructed by using Proposition 3.1 and Corollary 3.3 is the Ewens measure, which, for $E=\mathbb{N}^{*}$, is studied in detail in [13]. The precise existence result in our more general setting is the following:

Proposition 3.4. Let $\theta$ be in $\mathbb{R}_{+}$and $E$ a countable set. For all $I \in \mathcal{F}(E)$, let $\mathbb{P}_{I}^{(\theta)}$ be the Ewens measure of parameter $\theta$, defined as follows: for all $\sigma \in \Sigma_{I}$,

$$
\mathbb{P}_{I}^{(\theta)}(\sigma)=\frac{\theta^{n-1}}{(\theta+1) \ldots(\theta+N-1)}
$$

where $n$ is the number of cycles of $\sigma$ and $N$ the cardinal of $I$. Then the family $\left(\mathbb{P}_{I}^{(\theta)}\right)_{I \in \mathcal{F}(E)}$ is compatible in the sense of Proposition [3.1: the measure $\mathbb{P}_{E}^{(\theta)}$ on $\left(\Sigma_{E}, \mathcal{S}_{E}\right)$ which is deduced from Corollary 3.3 is called the Ewens measure of parameter $\theta$.

The Ewens measures are particular cases of the so-called central measures. Indeed, for all countable sets $E$ and for all $g \in \Sigma_{E}^{(0)}$, the conjugation by $g$ defined in Proposition [2.6] is measurable with respect to the $\sigma$-algebra $\mathcal{S}_{E}$. Therefore it defines a group action on the set of probability measures on $\left(\Sigma_{E}, \mathcal{S}_{E}\right)$ : by definition, a probability measure $\mathbb{P}$ is central if and only if it is invariant by this action. It is easy to check that this condition holds if and only if all the image measures of $\mathbb{P}$ by the coordinate maps are invariant by conjugation (which is clearly the case for the Ewens measures). In the case of $E=\mathbb{N}^{*}$, Tsilevich (in [14]) has completely characterized the central measures, by using the properties of the partitions of countable sets, described by Kingman (see [4], [5], [6]). If the cardinality of a finite set $I$ is denoted by $|I|$, then the result by Tsilevich can easily be translated in our framework as follows:

Proposition 3.5. Let $E$ be a countable set, $\mathbb{P}$ a central measure on $\left(\Sigma_{E}, \mathcal{S}_{E}\right)$, and $\sigma$ a random virtual permutation following the distribution $\mathbb{P}$. Then, for all $x, y \in E$, the indicator of the event $\left\{x \sim_{\sigma} y\right\}$ is measurable with respect to the $\sigma$-algebra $\mathcal{S}_{E}$, and there exists a family of random variables $(\lambda(x))_{x \in E}$, unique up to almost sure equality, taking their values in the interval $[0,1]$, and satisfying the following properties:

- For $I \in \mathcal{F}(E)$,

$$
\frac{\left|I \cap \mathcal{C}_{\sigma}(x)\right|}{|I|} \underset{|I| \rightarrow \infty}{\longrightarrow} \lambda(x)
$$

in $L^{1}$, and then in all the spaces $L^{p}$ for $p \in[1, \infty)$ since all the variables involved here are bounded by one. 
- For any strictly increasing sequence $\left(I_{n}\right)_{n \geq 1}$ of nonempty sets in $\mathcal{F}(E)$,

$$
\frac{\left|I_{n} \cap \mathcal{C}_{\sigma}(x)\right|}{\left|I_{n}\right|} \underset{n \rightarrow \infty}{\longrightarrow} \lambda(x)
$$

almost surely.

- Almost surely, for all $x \in E, \lambda(x)=0$ if and only if $x$ is a fixed point of $\sigma$, i.e. a fixed point of $\sigma_{I}$ for all $I \in \mathcal{F}(E)$ containing $x$.

- Almost surely, for all $x, y \in E, \lambda(x)=\lambda(y)$ if $x \sim_{\sigma} y$.

For all $k \geq 1$, let us then define $\lambda_{k}$ as the supremum of $\inf _{1 \leq j \leq k} \lambda\left(x_{j}\right)$ for a sequence $\left(x_{j}\right)_{1 \leq j \leq k}$ of elements in $E$ satisfying $x_{j} \chi_{\sigma} x_{j^{\prime}}$ for $j \neq j^{\prime}$, the supremum of the empty set being taken to be equal to zero. The sequence $\left(\lambda_{k}\right)_{k \geq 1}$ is a random variable with values in the space of the sequences of elements in $[0,1]$, endowed with the $\sigma$-algebra generated by the coordinate maps, $\left(\lambda_{k}\right)_{k \geq 1}$ is uniquely determined up to almost sure equality, and it lies almost surely in the (measurable) simplex $\Lambda$ of non-increasing random sequences in $[0,1]$, satisfying the inequality:

$$
\sum_{k \geq 1} \lambda_{k} \leq 1 .
$$

Moreover, the central measure $\mathbb{P}$ is uniquely determined by the distribution of the sequence $\left(\lambda_{k}\right)_{k \geq 1}$, as follows:

- For any probability measure $\nu$ on the space $\Lambda$ endowed with the $\sigma$-algebra of the coordinate maps, there exists a unique central measure $\mathbb{P}_{\nu}$ on $\left(\Sigma_{E}, \mathcal{S}_{E}\right)$ such that the corresponding random sequence $\left(\lambda_{k}\right)_{k \geq 1}$ has distribution $\nu$.

- For all sequences $\lambda \in \Lambda$, let $\mathbb{P}_{\lambda}:=\mathbb{P}_{\delta_{\lambda}}$, where $\delta_{\lambda}$ denotes the Dirac measure at $\lambda$. Then, for all $A \in \mathcal{S}(E)$, the map $\lambda \mapsto \mathbb{P}_{\lambda}(A)$ is measurable and for all probability measures $\nu$, one has:

$$
\mathbb{P}_{\nu}(A)=\int_{\Lambda} \mathbb{P}_{\lambda}(A) d \nu(\lambda) .
$$

Remark 3.6. Intuitively, $\lambda(x)$ represents the asymptotic length of the cycle of $x$ and $\left(\lambda_{k}\right)_{k \geq 1}$ is the non-increasing sequence of cycle lengths. For all $\theta \geq 0$, the Ewens measure of parameter $\theta$ is equal to $\mathbb{P}_{\nu^{(\theta)}}$, where $\nu^{(\theta)}$ is the Poisson-Dirichlet distribution of parameter $\theta$ (Dirac measure at the sequence $(1,0,0, \ldots)$ if $\theta=0)$.

Proof. The measurability of the equivalence relation $\sim_{\sigma}$ is an immediate consequence of the fact that the event $x \sim_{\sigma} y$ depends only on the permutation $\sigma_{\{x, y\}}$. Therefore, $\pi_{0}: \sigma \mapsto \sim_{\sigma}$ is a measurable map from $\left(\Sigma_{E}, \mathcal{S}_{E}\right)$ to the space of equivalence relations of $E$, endowed with the $\sigma$-algebra generated by the events of the form $\{x \sim y\}$ for $x, y \in E$. Since this measurable space is canonically identified with a measurable space $\left(\Pi_{E}, \mathcal{V}_{E}\right)$ such that $\Pi_{E}$ is the space of partitions of the set $E$, the map $\pi_{0}$ can be identified to a measurable map $\pi$ from $\left(\Sigma_{E}, \mathcal{S}_{E}\right)$ to $\left(\Pi_{E}, \mathcal{V}_{E}\right)$. This map induces a map from the probability measures on $\left(\Sigma_{E}, \mathcal{S}_{E}\right)$ to the probability measures on $\left(\Pi_{E}, \mathcal{V}_{E}\right)$, and Proposition 2.6 implies that a central measure is always mapped to the distribution of an exchangeable partition. Let us now prove that this correspondence is bijective, i.e. for any distribution $\mathbb{Q}$ on $\left(\Pi_{E}, \mathcal{V}_{E}\right)$ inducing an exchangeable partition, there exists a unique central measure $\mathbb{P}$ on $\left(\Sigma_{E}, \mathcal{S}_{E}\right)$ such that the image of $\mathbb{P}$ by $\pi$ is equal to $\mathbb{Q}$. Indeed, this condition is satisfied if and only if for all $J \in \mathcal{F}(E)$, the law of the partition of $J$ induced by the cycle structure of $\sigma_{J}$, where $\left(\sigma_{I}\right)_{I \in \mathcal{F}(E)}$ follows the distribution 
$\mathbb{P}$, is equal to the law of the restriction to $J$ of a partition following the distribution $\mathbb{Q}$. Since a probability on $\Sigma_{J}$ which is invariant by conjugation is completely determined by the corresponding distribution of the cycle lengths, the uniqueness of $\mathbb{P}$ follows. Now, for all $J \in \mathcal{F}(E)$, and all distributions $\mathbb{Q}_{J}$ on the partitions of $J$, let us define $\pi^{-1}\left(\mathbb{Q}_{J}\right)$ as the distribution of a random permutation $\sigma_{J} \in \Sigma_{J}$ satisfying the following conditions:

- The partition of $J$ induced by the cycle structure of $\sigma_{J}$ follows the distribution $\mathbb{Q}_{J}$.

- Conditionally on this partition, the law of $\sigma_{J}$ is uniform.

The existence of a measure $\mathbb{P}$ satisfying the conditions above is then a consequence of the following property of compatibility, which can be easily checked: for all $I, J \in \mathcal{F}$ such that $I \subset J$, for all distributions $\mathbb{Q}_{J}$ on the partitions of $J$, the image of $\pi^{-1}\left(\mathbb{Q}_{J}\right)$ by the map from $\Sigma_{J}$ to $\Sigma_{I}$ which removes the elements of $J \backslash I$ from the cycle structure is equal to $\pi^{-1}\left(\mathbb{Q}_{I}\right)$, where $\mathbb{Q}_{I}$ is the image of $\mathbb{Q}_{J}$ by the restriction of the partitions to the set $I$. Now, the bijective correspondence induced by $\pi$ implies that it is sufficient to show the equivalent of Proposition 3.5 for exchangeable partitions. Let $\left(x_{n}\right)_{n \geq 1}$ be an enumeration of the set $E$, and let us define the strictly increasing family of sets $\left(I_{n}^{(0)}\right)_{n \geq 1}$, by:

$$
I_{n}^{(0)}:=\left\{x_{1}, x_{2}, \ldots, x_{n}\right\}
$$

If in Proposition 3.5 for exchangeable partitions, the convergence of $|I \cap \mathcal{C}(x)| /|I|$ to $\lambda(x)$ in $L^{1}$ when $|I|$ goes to infinity is removed, and if the almost sure convergence along any strictly increasing sequence of nonempty sets in $\mathcal{F}(E)$ is replaced by the convergence only along the sequence $\left(I_{n}^{(0)}\right)_{n \geq 1}$, then the remaining of the proposition is a direct consequence of classical results by Kingman. Let us now prove the convergence in $L^{1}$, fixing $x \in E$. By dominated convergence,

$$
\frac{\left|\mathcal{C}(x) \cap I_{n}^{(0)}\right|}{\left|I_{n}^{(0)}\right|} \underset{n \rightarrow \infty}{\longrightarrow} \lambda(x),
$$

in $L^{1}$ when $n$ goes to infinity. Moreover, for all $J, K \in \mathcal{F}(E)$ such that $\{x\} \subset J \subset K$, the joint law of $|\mathcal{C}(x) \cap J|$ and $|\mathcal{C}(x) \cap K|$ depends only on $|J|$ and $|K|$, since the underlying probability measure on $\left(\Sigma_{E}, \mathcal{S}_{E}\right)$ is central. Now, for $|J|$ large enough, $I_{|J|}^{(0)}$, and a fortiori $I_{|K|}^{(0)}$, contain $x$. In this case, one has

$$
\mathbb{E}\left[\left|\frac{|\mathcal{C}(x) \cap J|}{|J|}-\frac{|\mathcal{C}(x) \cap K|}{|K|}\right|\right]=\mathbb{E}\left[\left|\frac{\left|\mathcal{C}(x) \cap I_{|J|}^{(0)}\right|}{\left|I_{|J|}^{(0)}\right|}-\frac{\left|\mathcal{C}(x) \cap I_{|K|}^{(0)}\right|}{\left|I_{|K|}^{(0)}\right|}\right|\right] .
$$

By the convergence (3.1), the right-hand side of 3.2 tends to zero when $|J|$ goes to infinity. In other words, there exists a sequence $(\epsilon(n))_{n \geq 1}$, decreasing to zero at infinity, such that

$$
\mathbb{E}\left[\left|\frac{|\mathcal{C}(x) \cap J|}{|J|}-\frac{|\mathcal{C}(x) \cap K|}{|K|}\right|\right] \leq \epsilon(|J|),
$$

for all $J, K \in \mathcal{F}(E)$ such that $\{x\} \subset J \subset K$. Now, for all $J, K \in \mathcal{F}(E)$ containing $x$, we obtain, by taking $J \cup K$ as an intermediate set and by using the triangle inequality:

$$
\mathbb{E}\left[\left|\frac{|\mathcal{C}(x) \cap J|}{|J|}-\frac{|\mathcal{C}(x) \cap K|}{|K|}\right|\right] \leq \epsilon(|J|)+\epsilon(|K|) .
$$


Moreover, if $J \in \mathcal{F}(E)$ does not contain $x$, we have:

$$
\frac{|\mathcal{C}(x) \cap(J \cup\{x\})|}{|J \cup\{x\}|}-\frac{1}{|J|} \leq \frac{|\mathcal{C}(x) \cap J|}{|J|} \leq \frac{|\mathcal{C}(x) \cap(J \cup\{x\})|}{|J \cup\{x\}|} .
$$

Combining (3.3) and (3.4), we deduce, for any $J, K \in \mathcal{F}(E)$ :

$$
\mathbb{E}\left[\left|\frac{|\mathcal{C}(x) \cap J|}{|J|}-\frac{|\mathcal{C}(x) \cap K|}{|K|}\right|\right] \leq \epsilon(|J|)+\epsilon(|K|)+\frac{1}{|J|}+\frac{1}{|K|} .
$$

In particular, by taking $K=I_{n}^{(0)}$, one has for $n \geq|J|$ :

$$
\mathbb{E}\left[\left|\frac{|\mathcal{C}(x) \cap J|}{|J|}-\frac{\left|\mathcal{C}(x) \cap I_{n}^{(0)}\right|}{\left|I_{n}^{(0)}\right|}\right|\right] \leq 2 \epsilon(|J|)+\frac{2}{|J|},
$$

and, after letting $n \rightarrow \infty$ :

$$
\mathbb{E}\left[\left|\frac{|\mathcal{C}(x) \cap J|}{|J|}-\lambda(x)\right|\right] \leq 2 \epsilon(|J|)+\frac{2}{|J|}
$$

which proves the convergence in $L^{1}$. Now, let $\left(I_{n}\right)_{n \geq 1}$ be a strictly increasing sequence of sets in $\mathcal{F}(E)$, containing $x$ and such that $\left|I_{n}\right|=n$. Since the underlying measure on $\Sigma_{E}$ is central, the law of the sequence $\left(\left|\mathcal{C}(x) \cap I_{n}\right| / n\right)_{n \geq 1}$ is independent of the choice of $\left(I_{n}\right)_{n \geq 1}$. Hence, $\left(\left|\mathcal{C}(x) \cap I_{n}\right| / n\right)_{n \geq 1}$ is almost surely a Cauchy sequence, since it is the case when we suppose that $I_{n}=I_{n}^{(0)}$ for $n$ large enough. By the convergence in $L^{1}$ proven above, the limit of $\left(\left|\mathcal{C}(x) \cap I_{n}\right| / n\right)_{n \geq 1}$ is necessarily $\lambda(x)$ almost surely. The almost sure convergence is then proven for any strictly increasing sequence $\left(I_{n}\right)_{n \geq 1}$ such that $x \in I_{n}$ and $\left|I_{n}\right|=n$ for all $n \geq 1$ : the first condition can be removed simply by putting $x$ into all the sets $\left(I_{n}\right)_{n \geq 1}$, which changes the quotient $\left|\mathcal{C}(x) \cap I_{n}\right| /\left|I_{n}\right|$ by at most $1 /\left|I_{n}\right|$, the second one can be suppressed by inserting any strictly increasing sequence of nonempty sets in $\mathcal{F}(E)$ into an increasing sequence $\left(I_{n}\right)_{n \geq 1}$ such that $\left|I_{n}\right|=n$ for all $n \geq 1$.

As we have seen in its proof, the result of Proposition 3.5 gives some information about the asymptotic cycle lengths of a random virtual permutation following a central measure, but does not tell anything about the relative positions of the elements inside their cycle. It is a remarkable fact that these relative positions also admit an asymptotic limit when the size of the sets in $\mathcal{F}(E)$ goes to infinity:

Proposition 3.7. Let $E$ be a countable set, $x, y$ two elements of $E$ and $\sigma$ a virtual permutation of E, which follows a central probability measure. Then, on the event $\left\{x \sim_{\sigma} y\right\}$ :

- For all $I \in \mathcal{F}(E)$ containing $x$ and $y$, there exists a unique integer $k_{I}(x, y) \in$ $\left\{0,1, \ldots,\left|I \cap \mathcal{C}_{\sigma}(x)\right|-1\right\}$ such that $\sigma_{I}^{k}(x)=y$;

- The variable $k_{I}(x, y) /|I|$ converges in $L^{1}$, and then in all the spaces $L^{p}$ for $p \in[1, \infty)$, to a limit random variable $\Delta(x, y) \in[0,1]$ when $|I|$ goes to infinity

- For any strictly increasing sequence $\left(I_{n}\right)_{n \geq 1}$ of sets in $\mathcal{F}(E)$ containing $x$ and $y$, $k_{I_{n}}(x, y) /\left|I_{n}\right|$ converges almost surely to $\Delta(x, y)$ when $n$ goes to infinity.

Proof. Since Proposition 3.7 is obvious for $x=y$ (with $\Delta(x, x)=0$ ), let us suppose that $x \neq y$. If $x \sim y$, and if $I \in \mathcal{F}(E)$ contains $x$ and $y$, then these two elements of $E$ are in the same cycle of $\sigma_{I}$, which has length $\left|I \cap \mathcal{C}_{\sigma}(x)\right|$. This implies the existence and the uniqueness of $k_{I}(x, y)$. Now, let $\left(I_{n}\right)_{n \geq 1}$ be a strictly increasing sequence of sets in $\mathcal{F}(E)$ 
such that $I_{1}=\{x, y\}$, and for all $n \geq 1, I_{n+1}=I_{n} \cup\left\{z_{n}\right\}$ with $z_{n} \notin I_{n}$. Then, for all $n \geq 1$, conditionally on $\sigma_{I_{n}}$, and on the event $x \sim y \sim z_{n}$, the position of $z_{n}$ inside the cycle structure of $\sigma_{I_{n+1}}$ is uniform among all the possible positions in the cycle containing $x$ and $y$, since the $\sigma$ follows a central probability measure. Hence, again conditionally on $\sigma_{I_{n}}$, $x \sim y \sim z_{n}$, one has $k_{I_{n+1}}(x, y)=k_{I_{n}}(x, y)+1$ with probability $k_{I_{n}}(x, y) /\left|\mathcal{C}(x) \cap I_{n}\right|$ (if $z_{n}$ is inserted between $x$ and $y$ in their common cycle), and $k_{I_{n+1}}(x, y)=k_{I_{n}}(x, y)$ with probability $1-\left(k_{I_{n}}(x, y) /\left|\mathcal{C}(x) \cap I_{n}\right|\right)$. One deduces that $\left(k_{I_{n}}(x, y) \mathbb{1}_{x \sim y} /\left|\mathcal{C}(x) \cap I_{n}\right|\right)_{n \geq 1}$ is a martingale with respect to the filtration generated by $\left(\sigma_{I_{n}}\right)_{n \geq 1}$. Since it takes its values in $[0,1]$, it converges almost surely to a limit random variable. Moreover, on the event $\{x \sim y\}$, the quotient $\left|\mathcal{C}(x) \cap I_{n}\right| /\left|I_{n}\right|$ converges almost surely to $\lambda(x)$, which is almost surely strictly positive, since $x$ is not a fixed point of $\sigma$. Hence, again on the set $x \sim y, k_{I_{n}}(x, y) /\left|I_{n}\right|$ converges to a random variable $\Delta(x, y)$ almost surely, and then in $L^{1}$ by dominated convergence. Now, let $J, K$ be two finite subsets of $E$ containing $x$ and $y$. If $J$ is included in $K$, then by the $L^{1}$ convergence of $k_{I_{n}}(x, y) /\left|I_{n}\right|$ at infinity, and by the centrality of the law of $\sigma$, one has:

$$
\begin{aligned}
\mathbb{E}\left[\left|\left(k_{J}(x, y) /|J|\right)-\left(k_{K}(x, y) /|K|\right)\right| \mathbb{1}_{x \sim y}\right] & =\mathbb{E}\left[\left|\left(k_{I_{|J|-1}}(x, y) /|J|\right)-\left(k_{I_{|K|-1}}(x, y) /|K|\right)\right| \mathbb{1}_{x \sim y}\right] \\
& \leq \epsilon(|J|),
\end{aligned}
$$

where $\epsilon(n)$ decreases to zero at infinity. If $J$ is not supposed to be included in $K$, then by using $J \cup K$ as an intermediate step, one obtains:

$$
\mathbb{E}\left[\left|\left(k_{J}(x, y) /|J|\right)-\left(k_{K}(x, y) /|K|\right)\right| \mathbb{1}_{x \sim y}\right] \leq \epsilon(|J|)+\epsilon(|K|) .
$$

In particular, for $J \in \mathcal{F}(E)$ and for all $n \geq|J|$ :

$$
\mathbb{E}\left[\left|\left(k_{J}(x, y) /|J|\right)-\left(k_{I_{n}}(x, y) /\left|I_{n}\right|\right)\right| \mathbb{1}_{x \sim y}\right] \leq 2 \epsilon(|J|),
$$

which implies that

$$
\mathbb{E}\left[\left|\left(k_{J}(x, y) /|J|\right)-\Delta(x, y)\right| \mathbb{1}_{x \sim y}\right] \leq 2 \epsilon(|J|) .
$$

Therefore, on the set $x \sim y, k_{J}(x, y) /|J|$ converges in $L^{1}$ to $\Delta(x, y)$. Now, let $\left(J_{n}\right)_{n \geq 1}$ be a strictly increasing sequence of sets in $\mathcal{F}(E)$, containing $x$ and $y$. This sequence can be inserted in a sequence with satisfies the same assumptions as $\left(I_{n}\right)_{n \geq 1}$, which implies that on the event $\{x \sim y\}, k_{J_{n}}(x, y) /\left|J_{n}\right|$ converges almost surely to a random variable, necessarily equal to $\Delta(x, y)$ since $k_{J_{n}}(x, y) /\left|J_{n}\right|$ converges to $\Delta(x, y)$ in $L^{1}$.

For $x \sim y, \Delta(x, y)$ represents the asymptotic number of iterations of the virtual permutation $\sigma$ which are needed to go from $x$ to $y$, and $\Delta(x, y) / \lambda(x)$ is the asymptotic proportion of the cycle $\mathcal{C}(x)$, lying between $x$ and $y$. The distribution of this proportion is deduced from the following result:

Proposition 3.8. Let $E$ be a countable set, and let $\sigma$ be a virtual permutation of $E$, which follows a central probability measure. For $m, n_{1}, \ldots n_{m} \geq 0$, let $\left(x_{j}\right)_{1 \leq j \leq m}$ and $\left(y_{j, k}\right)_{1 \leq j \leq m, 1 \leq k \leq n_{j}}$ be distinct elements of $E$. Then, conditionally of the event:

$$
\left\{\forall j \in\{1, \ldots, m\}, \forall k \in\left\{1, \ldots, n_{j}\right\}, x_{j} \sim_{\sigma} y_{j, k}\right\} \cap\left\{\forall j_{1} \neq j_{2} \in\{1, \ldots, m\}, x_{j_{1}} \not x_{j_{2}}\right\},
$$

the variables $\left(\Delta\left(x_{j}, y_{j, k}\right) / \lambda\left(x_{j}\right)\right)_{1 \leq j \leq m, 1 \leq k \leq n_{j}}$ are almost surely well-defined, independent and uniform on $[0,1]$, and they form a family which is independent of the variables $(\lambda(x))_{x \in E}$.

Remark 3.9. It is possible to take some indexes $j$ such that $n_{j}=0$. In this case, there is no point of the form $y_{j, k}$, and then no variable of the form $\Delta\left(x_{j}, y_{j, k}\right) / \lambda\left(x_{j}\right)$, involved in Proposition 3.8 . 
Proof. Let $q \geq 1$, and let $\left(x_{j}\right)_{m+1 \leq j \leq m+q}$ be a family of elements of $E$ such that all the elements $\left(x_{j}\right)_{1 \leq j \leq m+q}$ are distinct. Let us denote:

$$
\mathcal{E}:=\left\{\forall j \in\{1, \ldots, m\}, \forall k \in\left\{1, \ldots, n_{j}\right\}, x_{j} \sim_{\sigma} y_{j, k}\right\} \cap\left\{\forall j_{1} \neq j_{2} \in\{1, \ldots, m\}, x_{j_{1}} \not x_{j_{2}}\right\} .
$$

Moreover, for all $I \in \mathcal{F}(E)$ containing $x_{j}$ for all $j \in\{1, \ldots, m+q\}$ and $y_{j, k}$ for all $j \in$ $\{1, \ldots, m\}, k \in\left\{1, \ldots n_{j}\right\}$, and for all sequences $\left(p_{j}\right)_{1 \leq j \leq m+q}$ of strictly positive integers such that $p_{j}>n_{j}$ for all $j \in\{1, \ldots, m\}$, let us define the following event:

$$
\mathcal{E}_{I,\left(p_{j}\right)_{1 \leq j \leq m}}:=\mathcal{E} \cap\left\{\forall j \in\{1, \ldots m+q\},\left|\mathcal{C}\left(x_{j}\right) \cap I\right|=p_{j}\right\} .
$$

By the centrality of the law of $\sigma$, conditionally on $\mathcal{E}_{I,\left(p_{j}\right)_{1 \leq j \leq m+q}}$, the sequences $\left(k_{I}\left(x_{j}, y_{j, k}\right)\right)_{1 \leq k \leq n_{j}}$, for $j \in\{1, \ldots, m\}$ such that $n_{j}>0$, are independent, and the law of $\left(k_{I}\left(x_{j}, y_{j, k}\right)\right)_{1 \leq k \leq n_{j}}$ is uniform among all the possible sequences of $n_{j}$ distinct elements in $\left\{1,2, \ldots, p_{j}-1\right\}$. Now, for all $j \in\{1, \ldots, m\}$, let $\Phi_{j}$ be a continuous and bounded function from $\mathbb{R}^{n_{j}}$ to $\mathbb{R}\left(\Phi_{j}\right.$ is a real constant if $\left.n_{j}=0\right)$. Since for $n_{j}>0$, the uniform distribution on the sequences of $n_{j}$ district elements in $\{1 / p, 2 / p, \ldots,(p-1) / p\}$ converges weakly to the uniform distribution on $[0,1]^{n_{j}}$ when $p$ goes to infinity, one has:

$$
\begin{aligned}
& \mathbb{E}\left[\prod_{j=1}^{m} \Phi_{j}\left(\left(k_{I}\left(x_{j}, y_{j, k}\right) / p_{j}\right)_{1 \leq k \leq n_{j}}\right) \mid \mathcal{E}_{I,\left(p_{j}\right)_{1 \leq j \leq m+q}}\right] \\
& =\prod_{j=1}^{m} \mathbb{E}\left[\Phi_{j}\left(\left(k_{I}\left(x_{j}, y_{j, k}\right) / p_{j}\right)_{1 \leq k \leq n_{j}}\right) \mid \mathcal{E}_{I,\left(p_{j}\right)_{1 \leq j \leq m+q}}\right] \\
& =\left(\prod_{j=1}^{m} \int_{[0,1]^{n_{j}}} \Phi_{j}\right)+\alpha\left(\left(\Phi_{j}, p_{j}\right)_{1 \leq j \leq m}\right),
\end{aligned}
$$

where for fixed functions $\left(\Phi_{j}\right)_{1 \leq j \leq m},\left|\alpha\left(\left(\Phi_{j}, p_{j}\right)_{1 \leq j \leq m}\right)\right|$ is uniformly bounded and tends to zero when the minimum of $p_{j}$ for $n_{j}>0$ goes to infinity. One deduces that for all continuous, bounded functions $\Psi$ from $\mathbb{R}^{m}$ to $\mathbb{R}$ :

$$
\begin{aligned}
& \mathbb{E}[\Psi\left.\left(\left(\frac{\left|\mathcal{C}\left(x_{j}\right) \cap I\right|}{|I|}\right)_{1 \leq j \leq m+q}\right) \prod_{j=1}^{m} \Phi_{j}\left(\left(k_{I}\left(x_{j}, y_{j, k}\right) /\left(\left|\mathcal{C}\left(x_{j}\right) \cap I\right|\right)\right)_{1 \leq k \leq n_{j}}\right) \mid \mathcal{E}\right] \\
&= \mathbb{E}\left[\Psi\left(\left(\frac{\left|\mathcal{C}\left(x_{j}\right) \cap I\right|}{|I|}\right)_{1 \leq j \leq m+q} \mid \mathcal{E}\right)\right]\left(\prod_{j=1}^{m} \int_{[0,1]^{n_{j}}} \Phi_{j}\right) \\
&+\mathbb{E}\left[\Psi\left(\left(\frac{\left|\mathcal{C}\left(x_{j}\right) \cap I\right|}{|I|}\right)_{1 \leq j \leq m+q}\right) \alpha\left(\left(\Phi_{j},\left|\mathcal{C}\left(x_{j}\right) \cap I\right|\right)_{1 \leq j \leq m}\right) \mid \mathcal{E}\right] .
\end{aligned}
$$

Now, for any strictly increasing sequence $\left(I_{r}\right)_{r \geq 1}$ of sets satisfying the same assumptions as $I$, and on the event $\mathcal{E}$ :

- For all $j \in\{1, \ldots, m+q\},\left|\mathcal{C}\left(x_{j}\right) \cap I_{r}\right| /\left|I_{r}\right|$ converges almost surely to $\lambda\left(x_{j}\right)$ when $r$ goes to infinity.

- For all $j \in\{1, \ldots, m\}$ and $k \in\left\{1, \ldots, n_{j}\right\}, k_{I_{r}}\left(x_{j}, y_{j, k}\right) /\left(\left|\mathcal{C}\left(x_{j}\right) \cap I_{r}\right|\right)$ tends almost surely to $\Delta\left(x_{j}, y_{j, k}\right) /\left(\lambda\left(x_{j}\right)\right)$, which is well-defined since $\lambda\left(x_{j}\right)>0$ almost surely (by assumption, $x_{j}$ is not a fixed point of $\sigma$ if $n_{j}>0$ ). 
- For all $j \in\{1, \ldots, m\}$ such that $n_{j}>0$, and then $\lambda\left(x_{j}\right)>0$ almost surely, $\left|\mathcal{C}\left(x_{j}\right) \cap I_{r}\right|$ tends almost surely to infinity, which implies that $\alpha\left(\left(\Phi_{j},\left|\mathcal{C}\left(x_{j}\right) \cap I\right|\right)_{1 \leq j \leq m}\right)$ goes to zero.

By dominated convergence, one deduces:

$$
\begin{gathered}
\left.\mathbb{E}\left[\Psi\left(\left(\lambda\left(x_{j}\right)\right)_{1 \leq j \leq m+q}\right) \prod_{j=1}^{m} \Phi_{j}\left(\Delta\left(x_{j}, y_{j, k}\right) /\left(\lambda\left(x_{j}\right)\right)\right)_{1 \leq k \leq n_{j}}\right) \mid \mathcal{E}\right] \\
=\mathbb{E}\left[\Psi\left(\left(\lambda\left(x_{j}\right)\right)_{1 \leq j \leq m+q}\right) \mid \mathcal{E}\right]\left(\prod_{j=1}^{m} \int_{[0,1]^{n_{j}}} \Phi_{j}\right) .
\end{gathered}
$$

This proves Proposition 3.8 with $(\lambda(x))_{x \in E}$ replaced by $\lambda\left(x_{j}\right)_{j \in\{1, \ldots, m+q\}}$. By increasing $q$ and by using monotone class theorem, we are done.

A particular case of Proposition 3.8 is the following:

Corollary 3.10. Let $E$ be a countable set, $x$ and $y$ two distinct elements of $E$, and $\sigma a$ virtual permutation of $E$, which follows a central probability measure. Then, conditionally on the event $\left\{x \sim_{\sigma} y\right\}$, the variable $\Delta(x, y) / \lambda(x)$ is almost surely well-defined and uniform on the interval $[0,1]$ (which implies that $\Delta(x, y) \in(0, \lambda(x))$ almost surely).

Recall now that by definition, for $x \sim y, I \in \mathcal{F}(E)$ containing $x$ and $y, k_{I}(x, y)$ is the unique integer between 0 and $|\mathcal{C}(x) \cap I|-1$ such that $\sigma^{k_{I}(x, y)}(x)=y$. If the condition $0 \leq k_{I}(x, y) \leq|\mathcal{C}(x) \cap I|-1$ is removed, then $k_{I}(x, y)$ is only defined up to a multiple of $|\mathcal{C}(x) \cap I|$. Hence, by taking $|I| \rightarrow \infty$, it is natural to introduce the class $\delta(x, y)$ of $\Delta(x, y)$ modulo $\lambda(x)$. This class satisfies the following properties:

Proposition 3.11. Let $E$ be a countable set, $x, y, z$ three elements of $E$ (not necessarily distinct), and $\sigma$ a virtual permutation of $E$, which follows a central probability measure. Then, almost surely on the event $\left\{x \sim_{\sigma} y \sim_{\sigma} z\right\}$ :

- $\delta(x, x)=0$;

- $\delta(x, y)=-\delta(y, x)$;

- $\delta(x, y)+\delta(y, z)=\delta(x, z)$.

Proof. If $x=y=z$ and if $x$ is a fixed point of $\sigma$, then Proposition 3.11 is trivial (with all the values of $\delta$ equal to zero). Hence, we can suppose that $x$ is not a fixed point of $\sigma$. Let us now prove the third item, which implies immediately the first (by taking $x=y=z$ ) and then the second (by taking $x=z$ ). For any $I \in \mathcal{F}(E)$ containing $x, y$ and $z$, one has necessarily, on the event $\{x \sim y \sim z\}$ :

$$
\sigma_{I}^{k_{I}(x, y)+k_{I}(y, z)}(x)=\sigma_{I}^{k_{I}(x, z)}(x)=z,
$$

which implies

$$
k_{I}(x, y)+k_{I}(y, z)-k_{I}(x, z) \in\{0,|\mathcal{C}(x) \cap I|\}
$$

since $k_{I}(x, y), k_{I}(y, z)$ and $k_{I}(x, z)$ are in the set $\{0,1, \ldots,|\mathcal{C}(x) \cap I|-1\}$. Let $\left(I_{n}\right)_{n \geq 1}$ be a strictly increasing sequence of sets in $\mathcal{F}(E)$ containing $x, y$ and $z$ : the sequence

$$
\left(\frac{k_{I_{n}}(x, y)+k_{I_{n}}(y, z)-k_{I_{n}}(x, z)}{\left|\mathcal{C}(x) \cap I_{n}\right|}\right)_{n \geq 1}
$$


of elements in $\{0,1\}$ converges almost surely to $(\Delta(x, y)+\Delta(y, z)-\Delta(x, z)) /(\lambda(x))$ (recall that $\lambda(x)>0$ since $x$ is not a fixed point of $\sigma)$. One deduces that $\Delta(x, y)+\Delta(y, z)-\Delta(x, z)$ is almost surely equal to 0 or $\lambda(x)$, which implies that $\delta(x, y)+\delta(y, z)=\delta(x, z)$.

The properties of $\delta$ suggest the following representation of the cycle structure of $\sigma$ : for $x \in E$ which is not a fixed point of $\sigma$, one puts the elements of the cycle of $x$ for $\sim_{\sigma}$ into a circle of perimeter $\lambda(x)$, in a way such that for two elements $y, z \in \mathcal{C}(x), \delta(y, z)$ is the length, counted counterclockwise, of the circle arc between $x$ and $y$. In order to make the representation precise, we shall fix the asymptotic cycle lengths of $\sigma$, by only considering the central measures of the form $\mathbb{P}_{\lambda}$, for a given sequence $\lambda$ in the simplex $\Lambda$. When we make this assumption, we do not lose generality, since by Proposition 3.5, any central measure on $\Sigma_{E}$ can be written as a mixture of the measures of the form $\mathbb{P}_{\lambda}$ (i.e. an integral with respect to a probability measure $\nu$ on $\Lambda$ ), and we obtain the following result:

Proposition 3.12. Let $\lambda:=\left(\lambda_{k}\right)_{k \geq 1}$ be a sequence in the simplex $\Lambda$, let $E$ be a countable set, and let $\sigma=\left(\sigma_{I}\right)_{I \in \mathcal{F}(E)}$ be a virtual permutation of $E$, following the central probability measure $\mathbb{P}_{\lambda}$ defined in Proposition 3.5. For $k \geq 1$ such that $\lambda_{k}>0$, let $C_{k}$ be a circle of perimeter $\lambda_{k}$, the circles being pairwise disjoint, and for all $x, y \in C_{k}$, let $y-x \in \mathbb{R} / \lambda_{k} \mathbb{Z}$ be the length of the arc of circle from $x$ to $y$, counted counterclockwise and modulo $\lambda_{k}$. Let $L$ be a segment of length $1-\sum_{k \geq 1} \lambda_{k}$ (the empty set if $\sum_{k \geq 1} \lambda_{k}=1$ ), disjoint of the circles $C_{k}$, and let $C$ be the unions of all the circles $C_{k}$ and L. Let $\mu$ the uniform probability measure on $C$ (endowed with the $\sigma$-algebra generated by the Borel sets of $L$ and $C_{k}, k \geq 1$ ), i.e. the unique measure such that $\mu(A)$ is equal to the length of $A$, for any set $A$ equal to an arc of one of the circles $C_{k}$, or a segment included in L. Then, after enlarging, when necessary, the probability space on which $\sigma$ is defined, there exists a family $\left(X_{x}\right)_{x \in E}$ of i.i.d. random variables on the space $C$, following the law $\mu$, and such that the following conditions hold almost surely:

- For all $x \in E, X_{x} \in L$ if and only if $x$ is a fixed point of $\sigma$.

- For all $x, y$, distinct elements of $E, x \sim_{\sigma} y$ if and only if $X_{x}$ and $X_{y}$ are on the same circle $C_{k}$, and in this case, $\lambda(x)=\lambda(y)=\lambda_{k}$.

- For all $x, y$, distinct elements of $E$ such that $x \sim_{\sigma} y, \delta(x, y)$ is equal to $X_{y}-X_{x}$ modulo $\lambda(x)$.

Proof. After enlarging, when necessary, the underlying probability space, it is possible to define, for all $\lambda \in(0,1)$ such that there exist several consecutive indices $k$ satisfying $\lambda_{k}=\lambda$, a uniform random permutation $\tau_{\lambda}$ of the set of these indices, for all $k \geq 1$ such that $\lambda_{k}>0$, a uniform random variable $U_{k}$ on $C_{k}$, and for all $n \geq 1$, a uniform variable $V_{n}$ on $L$, in a way such that the virtual permutation $\sigma$, the variables $\left(U_{k}\right)_{k \geq 1},\left(V_{n}\right)_{n \geq 1}$ and all the permutations of the form $\tau_{\lambda}$ are independent. Now, let $\left(x_{n}\right)_{n \geq 1}$ be an enumeration of $E$. By the results on partitions by Kingman, it is almost surely possible to define a sequence $\left(k_{n}\right)_{n \geq 1}$ of integers by induction, as follows:

- If $x_{n}$ is a fixed point of $\sigma$, then $k_{n}=0$.

- If $x_{n}$ is equivalent to $x_{m}$ for some $m<n$, then $k_{n}=k_{m}$, independently of the choice of the index $m$.

- If $x_{n}$ is not a fixed point of $\sigma$ and is not equivalent to $x_{m}$ for any $m<n$, then $k_{n}$ is an integer such that $\lambda\left(x_{n}\right)=\lambda_{k_{n}}$, and if this condition does not determine $k_{n}$ uniquely, 
then this integer is chosen in a way such that $k_{n}$ is different from $k_{m}$ for all $m<n$ and $\tau_{\lambda\left(x_{n}\right)}\left(k_{n}\right)$ is as small as possible.

The random permutations of the form $\tau_{\lambda}$ are used in order to guarantee the symmetry between all the circles in $C$ which have the same perimeter. Moreover, one checks that for all $m, n \geq 1$ such that $x_{m}$ and $x_{n}$ are not fixed points of $\sigma, \lambda\left(x_{n}\right)=\lambda_{k_{n}}>0$, and $x_{m} \sim x_{n}$ if and only if $k_{m}=k_{n}$. Let us now define the variables $\left(X_{x_{n}}\right)_{n \geq 1}$ by induction, as follows:

- If $k_{n}=0$, then $X_{x_{n}}=V_{n}$.

- If $k_{n}>0$ and $k_{n} \neq k_{m}$ for all $m<n$, then $X_{x_{n}}=U_{k_{n}} \in C_{k_{n}}$.

- If $k_{n}>0$, if $k_{n}=k_{m}$ for some $m<n$, and if $m_{0}$ denotes the smallest possible value of $m$ satisfying this equality, then $X_{x_{n}}$ is the unique point of $C_{k_{n}}=C_{k_{m_{0}}}$ such that $X_{x_{n}}-U_{k_{m_{0}}}=\delta\left(x_{m_{0}}, x_{n}\right)$, modulo $\lambda_{k_{n}}=\lambda_{k_{m_{0}}}$.

For all $n \geq 1, X_{x_{n}} \in L$ if and only if $k_{n}=0$, otherwise, $X_{x_{n}} \in C_{k_{n}}$. Moreover, if $k_{m}=k_{n}>0$ for some $m, n \geq 1$, and if $m_{0}$ is the smallest index such that $k_{m}=k_{m_{0}}$, then modulo $\lambda_{k_{m}}$, almost surely,

$$
X_{x_{n}}-X_{x_{m}}=\left(X_{x_{n}}-U_{k_{m_{0}}}\right)-\left(X_{x_{m}}-U_{k_{m_{0}}}\right)=\delta\left(x_{m_{0}}, x_{n}\right)-\delta\left(x_{m_{0}}, x_{m}\right)=\delta\left(x_{m}, x_{n}\right) .
$$

One deduces that Proposition 3.12 is satisfied, provided that the variables $\left(X_{x_{n}}\right)_{n \geq 1}$ are independent and uniform on $C$. In order to show this fact, let us first observe that by Proposition [3.8, the following holds: for all $n \geq 1$, conditionally on the restriction of the equivalence relation $\sim$ to the set $\left\{x_{1}, \ldots, x_{n}\right\}$, the variables $\Delta\left(x_{m_{0}}, x_{m}\right) / \lambda\left(x_{m_{0}}\right)$, for $m_{0}<m \leq n$ and $x_{m_{0}} \sim x_{m}$, where $x_{m_{0}}$ is the element of its equivalence class with the smallest index, are uniform on [0,1], independent, and form a family which is independent of $\left(\lambda\left(x_{m}\right)\right)_{1 \leq m \geq n}$. Now, once the restriction of $\sim$ to $\left\{x_{1}, \ldots, x_{n}\right\}$ is given, the sequence $\left(k_{m}\right)_{1 \leq m \leq n}$ is uniquely determined by the sequence $\left(\lambda\left(x_{m}\right)\right)_{1 \leq m \geq n}$, and the permutations of the form $\tau_{\lambda}$ (which form a family independent of $\sigma$ ), and then it is independent of the family of variables $\Delta\left(x_{m_{0}}, x_{m}\right) / \lambda\left(x_{m_{0}}\right)=\Delta\left(x_{m_{0}}, x_{m}\right) / \lambda_{k_{m_{0}}}$ stated above. Since the restriction of $\sim$ to $\left\{x_{1}, \ldots, x_{n}\right\}$ is a function of the sequence $\left(k_{m}\right)_{1 \leq m \leq n}$, one deduces that conditionally on this sequence, the variables $\left(X_{x_{m}}\right)_{m \geq 1}$ are independent, $X_{x_{m}}$ being uniform on $C_{k_{m}}$ if $k_{m}>0$, and uniform on $L$ if $k_{m}=0$. It is now sufficient to prove that the variables $\left(k_{n}\right)_{n \geq 1}$ are independent and that for all $k \geq 1, \mathbb{P}\left[k_{n}=k\right]=\lambda_{k}$. By symmetry, for $k^{(1)}, \ldots, k^{(p)} \geq 1$, and for any distinct integers $n_{1}, \ldots, n_{p} \geq 1$, the probability that $k_{n_{j}}=k^{(j)}$ for all $j \in\{1, \ldots, p\}$, does not depend on $n_{1}, \ldots, n_{p}$ (note that the permutations $\tau_{\lambda}$ play a crucial role for this step of the proof of Proposition 3.12). Hence, for $m>p$,

$$
\mathbb{P}\left[\forall j \in\{1, \ldots, p\}, k_{j}=k^{(j)}\right]=\frac{(m-p) !}{m !} \mathbb{E}\left[\sum_{1 \leq n_{1} \neq \cdots \neq n_{p} \leq m} \mathbb{1}_{\forall j \in\{1, \ldots, p\}, k_{n_{j}}=k^{(j)}}\right],
$$

and then:

$$
\begin{aligned}
\mathbb{E}\left[\prod_{j=1}^{p} \frac{\left(\left|\left\{r \in\{1, \ldots m\}, k_{r}=k^{(j)}\right\}\right|-p\right)_{+}}{m}\right] & \leq \mathbb{P}\left[\forall j \in\{1, \ldots, p\}, k_{j}=k^{(j)}\right] \\
& \leq \mathbb{E}\left[\prod_{j=1}^{p} \frac{\left|\left\{r \in\{1, \ldots m\}, k_{r}=k^{(j)}\right\}\right|}{m-p}\right]
\end{aligned}
$$


By using Proposition 3.5 and dominated convergence for $m$ going to infinity, one deduces:

$$
\mathbb{P}\left[\forall j \in\{1, \ldots, p\}, k_{j}=k^{(j)}\right]=\prod_{j=1}^{p} \lambda_{k_{j}} .
$$

Now, we observe that it is possible to recover the virtual permutation $\sigma$ from the variables $\left(X_{x}\right)_{x \in E}$. More precisely, one has the following:

Proposition 3.13. Let us consider the setting and the notation of Proposition 3.12. For all $I \in \mathcal{F}(E)$ and $x \in I$, the element $\sigma_{I}(x)$ can almost surely be obtained as follows:

- If $X_{x} \in L$, then $\sigma_{I}(x)=x$.

- If for $k \geq 1, X_{x} \in C_{k}$, then $\sigma_{I}(x)=y$, where $X_{y}$ is the first point of the intersection of $C_{k}$ and the set $\left\{X_{z}, z \in I\right\}$, encountered by moving counterclockwise on $C_{k}$, starting just after $X_{x}$ (for example, if $X_{x}$ is the unique element of $C_{k} \cap\left\{X_{z}, z \in I\right\}$, then $x$ is a fixed point of $\left.\sigma_{I}\right)$.

Proof. If $x$ is a fixed point of $\sigma_{I}$, then either $X_{x} \in L$, or the unique $y \in I$ such that $X_{y}$ is on the same circle as $X_{x}$ is $x$ itself. If $x$ is not a fixed point of $\sigma_{I}$, then $X_{\sigma_{I}(x)}$ is on the same circle $C_{k}$ as $X_{x}$. Moreover, for any $y \in I$ different from $x$ and $\sigma_{I}(x)$, but in the same cycle of $\sigma_{I}$, one has $0<\Delta\left(x, \sigma_{I}(x)\right)<\Delta(x, y)$. Hence, there is no point of $C_{k} \cap\left\{X_{z}, z \in I\right\}$ on the open circle arc coming counterclockwise from $X_{x}$ to $X_{\sigma_{I}(x)}$.

A consequence of Proposition 3.12 and 3.13 is the following:

Corollary 3.14. Let $E$ be a countable set, $\lambda$ a sequence in the simplex $\Lambda, C$ the set constructed in Proposition 3.12, and $\left(X_{x}\right)_{x \in E}$ a sequence of i.i.d. random variables, uniform on $C$. For all $I \in \mathcal{F}(E)$, it is almost surely possible to define a permutation $\sigma_{I} \in \Sigma_{I}$, by the construction given in Proposition [3.13. Moreover, $\left(\sigma_{I}\right)_{I \in E}$ is almost surely a virtual permutation, and its distribution is the measure $\mathbb{P}_{\lambda}$ defined in Proposition 3.5.

Proof. The possibility to define almost surely $\sigma_{I}$ for all $I \in \mathcal{F}(E)$ comes from the fact that the points $\left(X_{x}\right)_{x \in E}$ are almost surely pairwise distinct. Moreover, since $\left(\sigma_{I}\right)_{I \in \mathcal{F}(E)}$ is a deterministic function of the sequence of points $\left(X_{x}\right)_{x \in E}$, its distribution is uniquely determined by the assumptions of Proposition 3.14. Since Propositions 3.12 and 3.13 give a particular setting on which $\left(\sigma_{I}\right)_{I \in \mathcal{F}(E)}$ is a virtual permutation following the distribution $\mathbb{P}_{\lambda}$, we are done.

Proposition 3.5 and Corollary 3.14 give immediately the following description of all the central measures on $\Sigma_{E}$ :

Corollary 3.15. Let $\nu$ be a probability measure on $\Lambda, \lambda$ a random sequence following the distribution $\nu$, and $C$ the random set constructed from the sequence $\lambda$ as in Proposition 3.12. Let $\left(X_{x}\right)_{x \in E}$ be a sequence of random points of $C$, independent and uniform conditionally on $\lambda$. For all $I \in \mathcal{F}(E)$, it is almost surely possible to define a permutation $\sigma_{I} \in \Sigma_{I}$, by the construction given in Proposition 3.13. Moreover, $\left(\sigma_{I}\right)_{I \in E}$ is almost surely a virtual permutation, and its distribution is the measure $\mathbb{P}_{\nu}$ defined in Proposition 3.5 .

This construction will be used in the next section, in order to study how $\sigma$ acts on the completion of the space $E$ with respect to a random metric related to $\delta$. 


\section{A Flow of transformations on a COMPLETion of $E$}

When one looks at the construction of the Ewens measures on the set of virtual permutations which is given at the end of Section 3, it is natural to consider the random metric given by the following proposition:

Proposition 4.1. Let $E$ be a countable set, and $\sigma$ a virtual permutation of $E$, which follows a central distribution. Let $d$ be a random function from $E^{2}$ to $\mathbb{R}_{+}$, almost surely defined as follows:

- If $x, y \in E$ and $x \chi_{\sigma} y$, then $d(x, y)=1$;

- If $x, y \in E$ and $x \sim_{\sigma} y$, then $d(x, y)=\inf \{|a|, \delta(x, y)=a(\bmod . \lambda(x))\}$.

Then, $d$ is almost surely a distance on $E$.

Proof. For all $x \in E, \delta(x, x)=0$ almost surely, which implies that $d(x, x)=0$. Conversely if $d(x, y)=0$ for $x, y \in E$, then $x \sim y$ and $\delta(x, y)=0$, which holds with strictly positive probability only for $x=y$. Finally if $x, y, z \in E$, then there are two cases:

- If $x, y, z$ are not equivalent, then $d(x, y)=1$ or $d(y, z)=1$, and $d(x, z) \leq 1$, which implies that $d(x, y)+d(y, z) \leq d(x, z)$.

- If $x \sim y \sim z$, then the triangle inequality holds because $\delta(x, z)=\delta(x, y)+\delta(y, z)$ almost surely.

Once the metric space $(E, d)$ is constructed, it is natural to embed it in another space which is better known.

Proposition 4.2. Let $E$ be a countable set, let $\sigma$ be a virtual permutation following a central measure, let $\left(\lambda_{k}\right)_{k \geq 1}$ be the non-increasing sequence of the asymptotic cycle lengths of $\sigma$, defined in Proposition 3.5, and let $C$ be the random space defined from $\left(\lambda_{k}\right)_{k \geq 1}$ as in Proposition 3.12. Moreover, let us define the random metric $D$ on $C$ as follows:

- If $x, y$ are in $C_{k}$ for the same value of $k$, then $D(x, y)=\inf \left\{|a|, x-y=a\left(\bmod . \lambda_{k}\right)\right\}$.

- If $x=y \in L, D(x, y)=0$.

- Otherwise, $D(x, y)=1$.

Then, if the probability space is sufficiently large to guarantee the existence of the random variables $\left(X_{x}\right)_{x \in E}$ described in Proposition 3.12, and if $K:=\left\{X_{x}, x \in E\right\}$, then the application $x \mapsto X_{x}$ is almost surely a bijective isometry from $(E, d)$ to $(K, D)$.

Proof. Since the variables $\left(X_{x}\right)_{x \in E}$ are almost surely pairwise disjoint, the map $\phi: x \mapsto X_{x}$ is almost surely bijective from $E$ to $K$. By comparing the definitions of $d$ and $D$ and by using the fact that $\delta(x, y)=X_{y}-X_{x}$ for $x \sim y$, we easily see that $\phi$ is isometric.

The isometry defined in Proposition 4.2 gives an intuitive idea on how the completion of $(E, d)$ looks like:

Proposition 4.3. Let us take the assumptions and the notation of Proposition 4.2 and let us define the random metric space $(\widehat{E}, \widehat{d})$ as the completion of $(E, d)$. Then the following properties hold almost surely: 
- The map $\phi$ can be extended in a unique way to a bijective and isometric map $\widehat{\phi}$ from $(\widehat{E}, \widehat{d})$ to $(H, D)$, where

$$
H:=(K \cap L) \cup \bigcup_{k \geq 1} C_{k}
$$

- There exists a unique extension $\widehat{\sim}_{\sigma}$ of the equivalence relation $\sim_{\sigma}$ to the set $\widehat{E}$, such that the set $\left\{(x, y) \in \widehat{E}^{2}, x \widehat{\sim}_{\sigma} y\right\}$ is closed in $\widehat{E}^{2}$, for the topology induced by the distance $\widehat{d}$.

- For all $x, y$ distinct in $\widehat{E}, x \widehat{\sim}_{\sigma} y$ if and only if $\widehat{\phi}(x)$ and $\widehat{\phi}(y)$ are on a common circle $C_{k}$, for some $k \geq 1$.

- There exists a unique map $\widehat{\lambda}$ from $\widehat{E}$ to $[0,1]$, extending $\lambda$ in a continuous way.

- For all $x \in \widehat{E}, k \geq 1, \widehat{\lambda}(x)=\lambda_{k}$ if $\widehat{\phi}(x) \in C_{k}$ and $\widehat{\lambda}(x)=0$ if $\widehat{\phi}(x) \in L$.

- There exists a unique map $\widehat{\delta}$ from the set $\left\{(x, y) \in \widehat{E}^{2}, x \widehat{\sim}_{\sigma} y\right\}$ to $H$, extending $\delta$ to a continuous map, for the topologies induced by the distances $\widehat{d}$ and $D$;

- For all $x, y$ distinct in $\widehat{E}$ such that $x \widehat{\sim}_{\sigma} y, \widehat{\delta}(x, y)=\widehat{\phi}(y)-\widehat{\phi}(x)$, modulo $\lambda_{k}$, if $\widehat{\phi}(x)$ and $\widehat{\phi}(y)$ are on the circle $C_{k}$.

Proof. Since, conditionally on $\left(\lambda_{k}\right)_{k \geq 1}$ the variables $\left(X_{x}\right)_{x \in E}$ are independent and uniform on $C$, the space $(K, D)$ is almost surely dense in $(H, D)$, which implies the first item. The description of the third item proves the existence of $\widehat{\sim}_{\sigma}$ : its uniqueness comes from the fact that $\sim_{\sigma}$ is defined on a dense subset of $\widehat{E}^{2}$. Similarly, the existence of $\widehat{\lambda}$ is deduced from the description given in the fifth item, and its uniqueness comes from the density of $E$ in $\widehat{E}$. Finally, the uniqueness of $\widehat{\delta}$ comes from the density of the set $\left\{x, y \in E, x \sim_{\sigma} y\right\}$ in the set $\left\{(x, y) \in \widehat{E}^{2}, x \widehat{\sim}_{\sigma} y\right\}$, and its existence is due to the continuity, for all $k \geq 1$, of the map $(x, y) \mapsto \widehat{\phi}(y)-\widehat{\phi}(x)$ from $\left\{(x, y) \in \widehat{E}^{2}, \widehat{\phi}(x), \widehat{\phi}(y) \in C_{k}\right\}$ to $\mathbb{R} / \lambda_{k} \mathbb{Z}$.

Remark 4.4. If $L$ is empty, which happens if and only if $\sum_{k \geq 1} \lambda_{k}=1$ (for example under Ewens measure of any parameter), then $H$ is equal to $C$. Otherwise, $H$ is the union of the circles included in $C$, and a discrete countable set.

From now, the notations $\widehat{\phi}, \widehat{\lambda}, \widehat{\delta}, \widehat{\sim}_{\sigma}$, will be replaced by $\phi, \lambda, \delta, \sim_{\sigma}$ (or $\sim$ ), since this simplification is consistent with the previous notation. Note that the map $\phi$ is not determined by $\sigma$, since it depends to the choice of the variables $\left(X_{x}\right)_{x \in E}$ in Proposition 3.12, which is not unique in general (for example, if $\lambda_{1}>0$ almost surely, and if $a \in \mathbb{R}$ is fixed, then one can replace each point $X_{x} \in C_{1}$ by the unique point $X_{x}^{\prime} \in C_{1}$ such that $X_{x}^{\prime}-X_{x}=a$, modulo $\lambda_{1}$ ). However, as stated in Proposition 4.3, the extensions of $\sim, \lambda$ and $\delta$ are almost surely uniquely determined. We now have all the ingredients needed to construct the flow of transformations on $\widehat{E}$ indicated in the title of this section.

Proposition 4.5. With the notation above and the assumptions of Proposition 4.2, there exists almost surely a unique family $\left(S^{\alpha}\right)_{\alpha \in \mathbb{R}}$ of bijective isometries of the set $\widehat{E}$, such that for all $x \in \widehat{E}, S^{\alpha}(x) \sim x$ and in the case where $x$ is not a fixed point of $\sigma, \delta\left(x, S^{\alpha}(x)\right)=\alpha$ modulo $\lambda(x)$. Almost surely, this family is given as follows:

- If $x$ is a fixed point of $\sigma$, then $S^{\alpha}(x)=x$.

- If $x$ is not a fixed point of $\sigma$, then $S^{\alpha}(x)$ is the unique point of $\widehat{E}$ such that $\phi\left(S^{\alpha}(x)\right)$ is on the same circle as $\phi(x)$, and $\phi\left(S^{\alpha}(x)\right)-\phi(x)=\alpha$ modulo $\lambda(x)$. 
Moreover, almost surely, $S^{\alpha+\beta}=S^{\alpha} S^{\beta}$ for all $\alpha, \beta \in \mathbb{R}$.

Proof. For all $x, y \in \widehat{E}, \alpha \in \mathbb{R}$, let us denote by $C(x, y, \alpha)$ the condition described as follows:

- If $x$ is a fixed point of $\sigma$, then $y=x$.

- If $x$ is not a fixed point of $\sigma$, then $x \sim y$ and $\phi(y)-\phi(x)=\alpha$, modulo $\lambda(x)$.

It is clear that the condition $C(x, y, \alpha)$ determines uniquely $y$ once $x$ and $\alpha$ are fixed, which proves that almost surely, the map $S^{\alpha}$ from $\widehat{E}$ to $\widehat{E}$ is well-defined for all $\alpha \in \mathbb{E}$, and that its explicit description is given in Proposition 4.5. By using this description, it is immediate to deduce that for all $\alpha, \beta \in \mathbb{R}, S^{\alpha}$ is isometric and $S^{\alpha+\beta}=S^{\alpha} S^{\beta}$. In particular, $S^{\alpha} S^{-\alpha}=S^{0}$ is the identity map of $\widehat{E}$, and then $S^{\alpha}$ is bijective.

Now, as written in the introduction, the flow $\left(S^{\alpha}\right)_{\alpha \in \mathbb{R}}$ can be seen as the limit, for large sets $I \in \mathcal{F}(E)$, of a power of $\sigma_{I}$, with exponent approximately equal to $\alpha|I|$. The following statement gives a rigorous meaning of this idea.

Proposition 4.6. Let us take the assumptions and the notation above, and let us define a sequence $\left(\alpha_{n}\right)_{n \geq 1}$ in $\mathbb{R}$, such that $\alpha_{n} / n$ tends to a limit $\alpha$ when $n$ goes to infinity. Then, for all $\epsilon>0$, there exist $C(\epsilon), c(\epsilon)>0$ such that for all $I \in \mathcal{F}(E)$ :

$$
\mathbb{P}\left[\exists x \in I, d\left(\sigma_{I}^{\alpha_{|I|}}(x), S^{\alpha}(x)\right) \geq \epsilon\right] \leq C(\epsilon) e^{-c(\epsilon)|I|} .
$$

Proof. Let us suppose $\alpha>0$. Let $x \in E$, let $I$ be a finite subset of $E$ containing $x$, and let $A$ be the set of $y \in I$ which are equivalent to $x$ but different from $x$. Then, by Proposition 3.8, conditionally on $A$, and on the event where this set is not empty, the variables $\delta(x, y) / \lambda(x)$ for $y \in A$ are independent, uniform on $\mathbb{R} / \mathbb{Z}$ and they from of family which is independent of $\lambda(x)$. Moreover, if $\left(b_{k}\right)_{k \in \mathbb{Z}}$ denotes the increasing family of the reals in the class of $\delta(x, y) / \lambda(x)$ modulo 1 , for some $y \in A \cup\{x\}$, with $b_{0}$ equal to zero, then $\delta\left(x, \sigma_{I}^{\alpha_{|I|}}(x)\right) / \lambda(x)$ is the class of $b_{\alpha_{|I|}}$ modulo 1 . One deduces that conditionally on $|A|$, this cardinality being different from zero,

$$
\delta\left(S^{\alpha}(x), \sigma_{I}^{\alpha_{|I|}}(x)\right)=\lambda(x) b\left(|A|, \alpha_{|I|}\right)-\alpha,
$$

where $(b(|A|, k))_{k \in \mathbb{Z}}$ is a 1-periodic random increasing family of reals, independent of $\lambda(x)$, and such that its elements in $[0,1)$ are $b(|A|, 0)=0$, and $|A|$ independent variables, uniform on $(0,1)$. One deduces that for $|A| \geq 1$,

$$
d\left(S^{\alpha}(x), \sigma_{I}^{\alpha_{|I|}}(x)\right) \leq\left|\lambda(x) b\left(|A|, \alpha_{|I|}\right)-\alpha\right| \wedge \lambda(x) .
$$

Moreover, one has obviously, in any case:

$$
d\left(S^{\alpha}(x), \sigma_{I}^{\alpha|I|}(x)\right) \leq \lambda(x) .
$$

One deduces, for all $\epsilon>0$ :

$$
\begin{aligned}
& \mathbb{P}\left[d\left(S^{\alpha}(x), \sigma_{I}^{\alpha_{|I|}}(x)\right) \geq \epsilon\right] \leq \mathbb{P}\left[\left|\frac{\alpha_{|I|}}{|I|}-\alpha\right| \geq \epsilon / 3\right]+\mathbb{P}\left[\lambda(x) \geq \epsilon,\left|\frac{\lambda(x) \alpha_{|I|}}{|A|}-\frac{\alpha_{|I|} \mid}{|I|}\right| \geq \epsilon / 3\right] \\
& \quad+\mathbb{P}\left[\lambda(x) \geq \epsilon,|A| \geq 1,\left|\frac{\lambda(x) \alpha_{|I|}}{|A|}-\alpha\right| \leq 2 \epsilon / 3, \lambda(x)\left|b\left(|A|, \alpha_{|I|}\right)-\frac{\alpha_{|I|} \mid}{|A|}\right| \geq \epsilon / 3\right] .
\end{aligned}
$$

In (4.1), the event involved in the second term of the sum is always supposed to occur if $A$ is empty. Now, for $|I|$ large enough,

$$
\mathbb{P}\left[\left|\frac{\alpha_{|I|}}{|I|}-\alpha\right|_{20} \geq \epsilon / 3\right]=0,
$$


since $\alpha_{n} / n$ is deterministic and tends to $\alpha$ when $n \geq 1$ goes to infinity. Moreover,

$$
\begin{aligned}
\mathbb{P}\left[\lambda(x) \geq \epsilon,\left|\frac{\lambda(x) \alpha_{|I|}}{|A|}-\frac{\alpha_{|I|} \mid}{|I|}\right| \geq \epsilon / 3\right] & =\mathbb{P}\left[\lambda(x) \geq \epsilon,|| A|-\lambda(x)| I|| \geq \frac{\epsilon|A||I|}{3\left|\alpha_{|I|}\right|}\right] \\
& \leq \mathbb{P}\left[\lambda(x) \geq \epsilon,|| A|-\lambda(x)| I|| \geq \frac{\epsilon^{2}|I|^{2}}{6\left|\alpha_{|I|}\right|}\right] \\
& +\mathbb{P}[\lambda(x) \geq \epsilon,|A| \leq|I| \epsilon / 2] \\
& \leq 2 \mathbb{P}\left[|| A|-\lambda(x)| I|| \geq \frac{\epsilon^{2}|I|}{6 \alpha+2 \epsilon+1}\right],
\end{aligned}
$$

if $|I|$ is large enough (depending only on the sequence $\left(\alpha_{n}\right)_{n \geq 1}$ ). Now, conditionally on $\lambda(x)$, $|A|$ is the sum of $|I|-1$ independent Bernoulli random variables, with parameter $\lambda(x)$. Hence, there exist $c_{1}, c_{2}>0$, depending only on $\left(\alpha_{n}\right)_{n \geq 1}$ and $\epsilon$, such that:

$$
\mathbb{P}\left[\lambda(x) \geq \epsilon,\left|\frac{\lambda(x) \alpha_{|I|}}{|A|}-\frac{\alpha_{|I|}}{|I|}\right| \geq \epsilon / 3\right] \leq c_{1} e^{-c_{2}|I|} .
$$

In order to evaluate the last term of (4.1), let us observe that if the corresponding event holds, then for $|I|$ large enough, $\alpha_{|I|}>|I| \alpha / 2$, which implies:

$$
|A| \geq \frac{\lambda(x) \alpha_{|I|}}{\alpha+2 \epsilon / 3} \geq \frac{\epsilon \alpha|I|}{2(\alpha+\epsilon)}
$$

Moreover, one has:

$$
b\left(|A|, \alpha_{|I|}\right)=\beta+\left[\alpha_{|I|} /(|A|+1)\right],
$$

where the brackets denote the integer part, and where, conditionally on $|A|, \beta$ is a beta random variable of parameters $k:=\alpha_{|I|}-(|A|+1)\left[\alpha_{|I|} /(|A|+1)\right]$ and $(|A|+1)-k$. One deduces that, conditionally on $|A|$, the probability that $\left|b\left(|A|, \alpha_{|I|}\right)-\alpha_{|I|} /(|A|+1)\right|$ is greater than or equal to $\epsilon / 6$ decreases exponentially with $|A|$, independently of $\alpha_{|I|}$. Moreover, for $|I|$ large enough, by (4.4):

$$
\left|\frac{\alpha_{|I|}}{|A|}-\frac{\alpha_{|I|}}{|A|+1}\right| \leq \frac{\alpha_{|I|}}{|A|^{2}} \leq \frac{5(\alpha+\epsilon)^{2}}{\epsilon^{2} \alpha|I|} \leq \epsilon / 7
$$

One deduces that there exist $c_{3}, c_{4}>0$, depending only on $\left(\alpha_{n}\right)_{n \geq 1}$ and $\epsilon$, such that:

$$
\mathbb{P}\left[\lambda(x) \geq \epsilon,\left|\frac{\lambda(x) \alpha_{|I|}}{|A|}-\alpha\right| \leq 2 \epsilon / 3, \lambda(x)\left|b\left(|A|, \alpha_{|I|}\right)-\frac{\alpha_{|I|}}{|A|}\right| \geq \epsilon / 3\right] \leq c_{3} e^{-c_{4}}|I|,
$$

By (4.2), (4.3), (4.5), there exist $c_{5}, c_{6}>0$ such that

$$
\mathbb{P}\left[d\left(S^{\alpha}(x), \sigma_{I}^{\alpha_{|I|}}(x)\right) \geq \epsilon\right] \leq c_{5} e^{-c_{6}|I|} .
$$

By adding these estimates for all $x \in I$, one deduces Proposition 4.6 for $\alpha>0$. The proof is exactly similar for $\alpha<0$. Now, let $\left(\alpha_{n}\right)_{n \geq 1}$ and $\left(\beta_{n}\right)_{n \geq 1}$ be two sequences such that $\alpha_{n} / n$ and $\beta_{n} / n$ tend to 1 . Then,

$$
\begin{aligned}
\sup _{x \in I} d\left(x, \sigma_{I}^{\alpha_{|I|}-\beta_{|I|}}(x)\right) & \leq \sup _{x \in I} d\left(x, S^{1}\left(\sigma_{I}^{-\beta_{|I|}}(x)\right)\right)+\sup _{x \in I} d\left(S^{1}\left(\sigma_{I}^{-\beta_{|I|}}(x)\right), \sigma_{I}^{\alpha_{|I|}-\beta_{|I|}}(x)\right) \\
& \leq \sup _{x \in I} d\left(S^{-1}(x), \sigma_{I}^{-\beta_{|I|}}(x)\right)+\sup _{x \in I} d\left(S^{1}(x), \sigma_{I}^{\alpha_{|I|}}(x)\right),
\end{aligned}
$$


since $S^{1}$ is an isometry of $\widehat{E}$ and $\sigma_{I}^{-\beta_{|I|}}$ is a bijection of $|I|$. One deduces that Proposition 4.6 holds also for $\alpha=0$.

Corollary 4.7. With the assumptions of Proposition [4.6, if $|I|$ goes to infinity, then the supremum of $d\left(\sigma_{I}^{\alpha_{|I|}}(x), S^{\alpha}(x)\right)$ for $x \in I$ converges to zero in probability, in $L^{p}$ for all $p \in[1, \infty)$, and almost surely along any deterministic, strictly increasing sequence $\left(I_{n}\right)_{n \geq 1}$ of sets in $\mathcal{F}(E)$. In particular, if $\left(x_{n}\right)_{n \geq 1}$ is a random sequence of elements in $E$, such that $x_{n} \in I_{n}$ for all $n \geq 1$ and $x_{n}$ converges almost surely to a random limit $x \in \widehat{E}$ when $n$ goes to infinity (this situation holds if $x \in E$ and $x_{n}=x$ for $n$ large enough), then $\sigma_{I_{n}}^{\alpha_{\left|I_{n}\right|}}\left(x_{n}\right)$ converges almost surely to $S^{\alpha}(x)$ when $n$ goes to infinity.

Proof. The convergence in probability is directly implied by Proposition 4.6, and it implies convergence in $L^{p}$ for all $p \in[1, \infty)$, since the distance is bounded by one. The almost sure convergence is proven by using Borel-Cantelli lemma.

We have now constructed a flow of transformations of $\widehat{E}$ and we have related it in a rigorous way to the iterations of $\sigma_{I}$ for large sets $I$. In Section 5, we interpret this flow as a flow of operators on a suitable random functional space, and we construct its infinitesimal generator, as discussed in the introduction.

\section{A FLOW OF OPERATORS ON A RANDOM FUNCTIONAL SPACE}

Let us now define the random functional space on which the flow of operators described below acts. We first take, as before, a random virtual permutation $\sigma$ on a countable set $E$, which follows a central probability measure. By the results given in the previous sections, the following events hold almost surely:

- The variables $\lambda(x)$ are well-defined, strictly positive for all $x \in E$ which are not fixed points of $\sigma$, and $\lambda(x)=\lambda(y)$ for all $x, y \in E$ such that $x \sim_{\sigma} y$.

- With the definitions of Proposition 3.5, the non-increasing sequence $\left(\lambda_{k}\right)_{k \geq 1}$ of the cycle lengths is an element of the simplex $\Lambda$.

- The quantity $\delta(x, y)$ exists for all $x, y \in E$ such that $x \sim_{\sigma} y$, and $\delta(x, x)=0$, $\delta(x, y)=-\delta(y, x), \delta(x, y)+\delta(y, z)=\delta(x, z)$ for all $x, y, z$ such that $x \sim_{\sigma} y \sim_{\sigma} z$.

- There exists a bijective isometry $\phi$ from $E$ to a dense subset of

$$
H:=L_{0} \cup \bigcup_{k \geq 1, \lambda_{k}>0} C_{k},
$$

where $C_{k}$ is a circle of perimeter $\lambda_{k}$, the set $L_{0}$ is empty if $\sum_{k \geq 1} \lambda_{k}=1$ and countable if $\sum_{k>1} \lambda_{k}<1$, and the union is disjoint, for the distances $d$ and $D$ defined similarly as in Propositions 4.1 and 4.2

- The map $\lambda$, the distance $d$ and the equivalence relation $\sim_{\sigma}$ extend in a unique continuous way to the completed space $\widehat{E}$ of $E$, for the distance $d$.

- The map $\delta$ extends in a unique continuous way to the set $\left\{(x, y) \in \widehat{E}^{2}, x \sim_{\sigma} y\right\}$.

- The isometry $\phi$ extends in a unique way to a bijective isometry from $(\widehat{E}, d)$ to $(H, D)$.

- For all distinct $x, y \in \widehat{E}, x \sim_{\sigma} y$ if and only if $\phi(x)$ and $\phi(y)$ are on the same circle included in $H$.

- For all distinct $x, y \in \widehat{E}$ such that $x \sim_{\sigma} y, \delta(x, y)=\phi(y)-\phi(x)$, modulo $\lambda(x)$. 
- There exists a unique flow $\left(S^{\alpha}\right)_{\alpha \in \mathbb{R}}$ of isometric bijections of $H$ such that for all $\alpha \in \mathbb{R}, x \in \widehat{E}, \phi\left(S^{\alpha}(x)\right)-\phi(x)=\alpha$ modulo $\lambda(x)$ if $x$ is not a fixed point of $\sigma$, and $S^{\alpha}(x)=x$ if $x$ is a fixed point of $\sigma$.

- For all $\alpha, \beta \in \mathbb{R}, S^{\alpha} S^{\beta}=S^{\alpha+\beta}$.

From now, let us fix $\sigma$ such that all the items above are satisfied: no randomness is involved in the construction of the operator $U$ given below. We can interpret the flow $\left(S^{\alpha}\right)_{\alpha \in \mathbb{R}}$ as a flow of operators on a functional space defined on $E$. The first step in the corresponding construction is the following result:

Proposition 5.1. Let $f$ be a function from $E$ to $\mathbb{C}$. If $f$ can be extended to a continuous function from $\widehat{E}$ to $\mathbb{C}$, then this extension is unique: in this case we say that $f$ is continuous. For example, if $f$ is uniformly continuous from $E$ to $\mathbb{C}$, then it is continuous and its continuous extension to $\widehat{E}$ is also uniformly continuous.

When a function from $E$ to $\mathbb{C}$ is continuous, we can use its extension to $\widehat{E}$ in order to make the flow $\left(S^{\alpha}\right)_{\alpha \in \mathbb{R}}$ acting on it, as follows:

Proposition 5.2. In the setting above, one can define a unique flow $\left(T^{\alpha}\right)_{\alpha \in \mathbb{R}}$ of linear operators on the space of continuous functions from $E$ to $\mathbb{C}$, satisfying the following properties:

- For any continuous function $f$ from $E$ to $\mathbb{C}, T^{\alpha}(f)(x)=\widehat{f}\left(S^{\alpha}(x)\right)$ for all $\alpha \in \mathbb{R}$, where $\widehat{f}$ is the continuous extension of $f$ to $\widehat{E}$;

- For all $\alpha, \beta \in \mathbb{R}, T^{\alpha+\beta}=T^{\alpha} T^{\beta}$.

Proof. Let $f, g, h$ be three continuous functions from $E$ to $\mathbb{C}$, such that $h=f+r g$ for some $r \in \mathbb{C}$. Let $\widehat{f}, \widehat{g}$ and $\widehat{h}$ be their continuous extensions to $\widehat{E}$. By uniqueness, one has $\widehat{h}=\widehat{f}+r \widehat{g}$. Let us now define, for $\alpha \in \mathbb{R}$, three functions $f_{\alpha}, g_{\alpha}$ and $h_{\alpha}$ from $\widehat{E}$ to $\mathbb{C}$, by:

$$
\begin{aligned}
& f_{\alpha}(x)=\widehat{f}\left(S^{\alpha}(x)\right), \\
& g_{\alpha}(x)=\widehat{g}\left(S^{\alpha}(x)\right),
\end{aligned}
$$

and

$$
h_{\alpha}(x)=\widehat{h}\left(S^{\alpha}(x)\right)
$$

for all $x \in \widehat{E}$. Since $S^{\alpha}$ is an isometry of $\widehat{E}$, the functions $f_{\alpha}, g_{\alpha}$ and $h_{\alpha}$ are continuous and satisfy: $h_{\alpha}=f_{\alpha}+r g_{\alpha}$. By taking their restrictions to $E$, one deduces the existence and the linearity of an operator $T^{\alpha}$, from the space of continuous functions on $E$ to itself, which satisfies the first item. Of course this operator is uniquely determined. Now, let $\beta \in \mathbb{R}$. One has, for all $x \in E$ :

$$
T^{\alpha} T^{\beta}(f)(x)=\widehat{T^{\beta}(f)}\left(S^{\alpha}(x)\right)
$$

and then,

$$
T^{\alpha} T^{\beta}(f)(x)=\widehat{f}\left(S^{\beta} S^{\alpha}(x)\right)=\widehat{f}\left(S^{\alpha+\beta}(x)\right)=T^{\alpha+\beta}(f)(x) .
$$

Let us now define the space of continuously differentiable functions with respect to the flow of operators $\left(T_{\alpha}\right)_{\alpha \in \mathbb{R}}$ : 
Definition 5.3. In the setting above, let $f$ be a continuous function from $E$ to $\mathbb{C}$. We say that $f$ is continuously differentiable, if and only if there exists a continuous function $U f$ from $E$ to $\mathbb{C}$, necessarily unique, such that for all $x \in \widehat{E}$,

$$
\frac{\widehat{T^{\alpha}(f)}(x)-\widehat{f}(x)}{\alpha} \underset{\alpha \rightarrow 0}{\longrightarrow} \widehat{U f}(x),
$$

where $\widehat{f}, \widehat{T^{\alpha}(f)}$ and $\widehat{U f}$ are the continuous extensions of $f, T^{\alpha}(f)$ and $U f$ to $\widehat{E}$.

The following result is immediate:

Proposition 5.4. The application $f \mapsto U f$ from the space of continuously differentiable functions to the space of continuous functions from $E$ to $\mathbb{C}$, constructed in Definition 5.3, is a linear operator.

Once the operator $U$ is defined, it is natural to study its eigenfunctions and eigenvalues. The following result holds:

Proposition 5.5. The eigenvalues of $U$ are zero, and all the nonzero multiples of $2 i \pi / \lambda_{k}$ for $\lambda_{k}>0$. The corresponding eigenspaces are described as follows:

- The space corresponding to the eigenvalue zero consists of all the functions $f$ from $E$ to $\mathbb{C}$ such that $x \sim_{\sigma} y$ implies $f(x)=f(y)$.

- The space corresponding to the eigenvalue ai for $a \in \mathbb{R}^{*}$ consists of all the functions $f$ such that $f(x)=0$ if $\lambda(x)=0$ or $\lambda(x)$ is not a multiple of $2 \pi / a$, and such that for $\lambda(x)$ nonzero and divisible by $2 \pi / a$, the restriction of $f$ to the equivalence class of $x$ for $\sim_{\sigma}$ is proportional to $y \mapsto e^{a i \delta(x, y)}$.

Consequently, the dimension of the space corresponding to the eigenvalue zero is equal to the number of indices $k \geq 1$ such that $\lambda_{k}>0$ if $\sum_{k \geq 1} \lambda_{k}=1$, and to infinity if $\sum_{k \geq 1} \lambda_{k}<1$. Moreover, for $a \in \mathbb{R}^{*}$, the dimension of the space corresponding to the eigenvalue $i$ ia is equal to the number of indices $k \geq 1$ such that $\lambda_{k}$ is a nonzero multiple of $2 \pi / a$, in particular it is finite.

Proof. Let $f$ be an eigenfunction of $U$ for an eigenvalue $b \in \mathbb{C}$, and let $\widehat{f}$ be its extension to $\widehat{E}$. One has $U f=b f$, and then by continuity, $\widehat{U f}=b \widehat{f}$. For all $x \in E$, let $g_{x}$ be the function from $\mathbb{R}$ to $\mathbb{C}$, given by:

$$
g_{x}(\alpha):=\widehat{f}\left(S^{\alpha}(x)\right)
$$

For all $\alpha, \beta \in \mathbb{R}$,

$$
g_{x}(\alpha+\beta)=\widehat{f}\left(S^{\alpha} S^{\beta}(x)\right)=\widehat{T^{\beta}(f)}\left(S^{\alpha}(x)\right)
$$

and then, for $\beta \neq 0$,

$$
\frac{g_{x}(\alpha+\beta)-g_{x}(\alpha)}{\beta}=\frac{\widehat{T^{\beta}(f)}\left(S^{\alpha}(x)\right)-\widehat{f}\left(S^{\alpha}(x)\right)}{\beta} \underset{\beta \rightarrow 0}{\longrightarrow} \widehat{U f}\left(S^{\alpha}(x)\right)=b \widehat{f}\left(S^{\alpha}(x)\right)=b g_{x}(\alpha) .
$$

Hence, $g_{x}$ is continuously differentiable and satisfies the differential equation $g_{x}^{\prime}=b g_{x}$, which implies that $g_{x}$ is proportional to the function $\alpha \rightarrow e^{b \alpha}$. Since $S^{\alpha}(x)=x$ for $\alpha=\lambda(x)$, and for all $\alpha \in \mathbb{R}$ if $\lambda(x)=0$, one has $\lambda(x)>0$ and $e^{b \lambda(x)}=1, b=0$ and $g_{x}$ constant, or $g_{x}$ identically zero. Therefore, one of the three following possibilities holds for all $x \in E$ :

- $f$ is identically zero on the cycle of $x$.

- $b=0$ and $f$ is constant on the cycle of $x$. 
- $\lambda(x)>0, b$ is multiple of $2 i \pi / \lambda(x)$ and the restriction of $f$ to the cycle of $x$ is proportional to $y \mapsto e^{b \delta(x, y)}$.

Conversely, it is easy to check that any function which satisfies one of the three items above for all $x \in E$ is an eigenfunction of $U$ for the eigenvalue $b$, which completes the proof of Proposition 5.5 .

As discussed before, the operator $T^{\alpha}$ can be viewed as a limit of $\sigma_{I}^{\alpha_{|I|}}$ for large $I \in \mathcal{F}(E)$ and $\alpha_{|I|}$ equivalent to $\alpha|I|$. It is then natural to relate the permutation $\sigma_{I}$ to the operator $T^{1 /|I|}$, and then the operator $|I|\left(\sigma_{I}-\mathrm{Id}\right)$ to $|I|\left(T^{1 /|I|}-\mathrm{Id}\right)$, where $\sigma_{I}$ is identified with a permutation matrix. Now, the eigenvalues of $|I|\left(\sigma_{I}-\mathrm{Id}\right)$ are equal to $|I|\left(e^{i \kappa}-1\right)$, where $\kappa$ is an eigenangle of $\sigma_{I}$, and this quantity is expected to be close to $i \kappa|I|$, on the other hand, $|I|\left(T^{1 /|I|}-\mathrm{Id}\right)$ is expected to be close to $U$. Hence, it is natural to compare the renormalized eigenangles of $\sigma_{I}$ (i.e. multiplied by $\left.i|I|\right)$, and the eigenvalues of $U$ computed in Proposition 5.5. The rigorous statement corresponding to this idea is the following:

Proposition 5.6. Let $\sigma$ be a random virtual permutation on a countable set E, following a central measure. Let $X$ be the set of the eigenvalues of the random operator $i U$ (which is almost surely well-defined), and for $I \in \mathcal{F}(E)$, let $X_{I}$ be the set of the eigenangles of $\sigma_{I}$, multiplied by $|I|$. If $\gamma \in X$ (resp. $\gamma \in X_{I}$ ), let $m(\gamma)$ (resp. $m_{I}(\gamma)$ ) be the multiplicity of the corresponding eigenvalue (resp. rescaled eigenangle). Then $X$ and $X_{I}, I \in \mathcal{F}(E)$, are included in $\mathbb{R}$, and for all continuous functions $f$ from $\mathbb{R}$ to $\mathbb{R}_{+}$, with compact support, the following convergence holds:

$$
\sum_{\gamma \in X_{I}} m_{I}(\gamma) f(\gamma) \underset{|I| \rightarrow \infty}{\longrightarrow} \sum_{\gamma \in X} m(\gamma) f(\gamma),
$$

in probability, and almost surely along any fixed, strictly increasing sequence of sets in $\mathcal{F}(E)$.

Proof. Since $X$ and $X_{I}$ have no point in the interval $(-2 \pi, 2 \pi)$ except zero, it is sufficient to prove the convergence stated in Proposition 5.6 for $f=\mathbb{1}_{\{0\}}$ and for $f$ nonnegative, continuous, with compact support and such that $f(0)=0$. Let $\left(I_{n}\right)_{n \geq 1}$ be an increasing sequence of subsets of $E$, such that $\left|I_{n}\right|=n$. Let us suppose that the underlying probability space is large enough to apply Proposition 3.12, and let us take the same notation. The multiplicity $m_{I_{n}}(0)$ is equal to the sum of the number of indices $k \geq 1$ such that there exists $x \in I_{n}$ with $X_{x} \in C_{k}$, and the number of elements $x \in I_{n}$ such that $X_{x} \in L$. By the fact that conditionally on $\left(\lambda_{k}\right)_{k \geq 1}$, the variables $\left(X_{x}\right)_{x \in E}$ are independent and uniform on $C$, a weak form of the law of large numbers implies that $m_{I_{n}}(0)$ increases almost surely to the number of indices $k \geq 1$ such that $\lambda_{k}>0$ if $\sum_{k \geq 1} \lambda_{k}=1$, and to infinity otherwise. In other words, $m_{I_{n}}(0)$ increases almost surely to $m(0)$, and then also in probability. Since the law of $\sigma$ is central, the convergence in probability holds also for $|I|$ going to infinity and not only along the sequence $\left(I_{n}\right)_{n \geq 1}$, which proves the convergence in Proposition 5.6 for $f=\mathbb{1}_{\{0\}}$. Let us now suppose that $f$ is nonnegative, continuous, with compact support and satisfies $f(0)=0$. One has almost surely, for all $n \geq 1$,

$$
\sum_{\gamma \in X_{I_{n}}} m(\gamma) f(\gamma)=\sum_{m \in \mathbb{Z} \backslash\{0\}} \sum_{k \geq 1} f\left(2 \pi m n /\left|I_{n} \cap \mathcal{C}_{k}\right|\right),
$$

where $\mathcal{C}_{k}$ denotes the set of $x \in E$ such that $X_{x} \in C_{k}$, and with the convention:

$$
f\left(2 \pi m n /\left|I_{n} \cap \mathcal{C}_{k}\right|\right):=0
$$


for $\left|I_{n} \cap \mathcal{C}_{k}\right|=0$. Since $f$ has compact support, there exists $A>0$ such that $f(t)=0$ for $|t| \geq A$. Hence, the condition $f\left(2 \pi m n /\left|I_{n} \cap \mathcal{C}_{k}\right|\right)>0$ implies that $2 \pi|m|<A$ and a fortiori $|m| \leq A$, on the other hand, it implies that $2 \pi n /\left|I_{n} \cap \mathcal{C}_{k}\right|<A,\left|I_{n} \cap \mathcal{C}_{k}\right| / n \geq 1 / A$, and in particular,

$$
\frac{1}{n}\left|I_{n} \cap\left(\bigcup_{l \geq k} \mathcal{C}_{l}\right)\right| \geq 1 / A
$$

Now, conditionally on $\left(\lambda_{n}\right)_{n \geq 1}$, the left hand side of (5.1) has the same law as the average of $n$ i.i.d. Bernoulli random variables, with parameter $\sum_{l \geq k} \lambda_{l}$. Hence, by law of large numbers, if $k_{0} \geq 1$ denotes the smallest integer such that $\sum_{l \geq k_{0}} \lambda_{l} \leq 1 / 2 A$, one has almost surely, for $n$ large enough, $f\left(2 \pi m n /\left|I_{n} \cap \mathcal{C}_{k}\right|\right)=0$ if $k \geq k_{0}$, and then

$$
\sum_{\gamma \in X_{I_{n}}} m(\gamma) f(\gamma)=\sum_{m \in(\mathbb{Z} \backslash\{0\}) \cap[-A, A]} \sum_{1 \leq k \leq k_{0}} f\left(2 \pi m n /\left|I_{n} \cap \mathcal{C}_{k}\right|\right) .
$$

By the continuity of $f$ and the fact that $\left|I_{n} \cap \mathcal{C}_{k}\right| / n$ tends to $\lambda_{k}$ when $n$ goes to infinity, one deduces that almost surely,

$$
\sum_{\gamma \in X_{I_{n}}} m(\gamma) f(\gamma) \underset{n \rightarrow \infty}{\longrightarrow} \sum_{m \in(\mathbb{Z} \backslash\{0\}) \cap[-A, A]} \sum_{1 \leq k \leq k_{0}} f\left(2 \pi m / \lambda_{k}\right)=\sum_{\gamma \in X} f(\gamma),
$$

which gives the almost sure convergence stated in Proposition 5.6. By the centrality of the law of $\sigma$, one then deduces the convergence in probability.

\section{REFERENCES}

1. A. Borodin and G. Olshanski, Infinite random matrices and ergodic measures, Comm. Math. Phys. 223 (2001), no. 1, 87-123.

2. S.-N. Evans, Eigenvalues of random wreath products, Electr. Jour. of Probab. 7 (2002), no. 9, 1-15.

3. S.-V. Kerov, G.-I. Olshanski, and A.-M. Vershik, Harmonic analysis on the infinite symmetric group, Comptes Rend. Acad. Sci. Paris 316 (1993), 773-778.

4. J.-F.-C. Kingman, Random discrete distribution, J. Roy. Stat. Soc. B 37 (1975), 1-22.

5. _ Random partitions in population genetics, Proc. R. Soc. Lond. (A) 361 (1978), 1-20.

6. The representation of partition structures, J. London Math. Soc. (2) 18 (1978), 374-380.

7. M.-L. Mehta, Random matrices, Pure and Applied Mathematics Series, Elsevier Academic Press, 2004.

8. J. Najnudel and A. Nikeghbali, The distribution of eigenvalues of randomized permutation matrices, http://arxiv.org/pdf/1005.0402, 2010.

9. Y.-A. Neretin, Hua type integrals over unitary groups and over projective limits of unitary groups, Duke Math. Jour. 114 (2002), 239-266.

10. G. Olshanski and A. Vershik, Ergodic unitarily invariant measures on the space of infinite Hermitian matrices, Amer. Math. Soc. Trans. 175 (1996), 137-175.

11. J. Pitman, Combinatorial Stochastic Processes, Lecture Notes in Math., vol. 1875, Springer-Verlag, Berlin, 2006.

12. J. Ramirez, B. Valkó, and B. Virág, Beta ensembles, stochastic Airy spectrum, and a diffusion, http://arxiv.org/pdf/math/0607331, 2006.

13. N.-V. Tsilevich, Distribution of cycle lengths of infinite permutations, Jour. of Math. Sciences 87 (1997), no. $6,4072-4081$.

14. Stationary measures on the space of virtual permutations for an action of the infinite symmetric group, 1998.

15. B. Valkó and B. Virág, Continuum limits of random matrices and the Brownian carousel, Inventiones Math. 177 (2009), 463-508. 
16. K. Wieand, Eigenvalue distributions of random permutation matrices, Ann. Probab. 28 (2000), no. 4, $1563-1587$.

Institut FÜr Mathematik, Universität ZürICh, WinterthURERStrasse 190, 8057-Zürich, SWITZERLAND

E-mail address: joseph.najnudel@math.uzh.ch

E-mail address: ashkan.nikeghbali@math.uzh.ch 